\title{
A weak Harnack inequality for fractional evolution equations with discontinuous coefficients
}

\author{
RICO ZACHER
}

\begin{abstract}
We study linear time fractional diffusion equations in divergence form of time order less than one. It is merely assumed that the coefficients are measurable and bounded, and that they satisfy a uniform parabolicity condition. As a main result we establish for nonnegative weak supersolutions of such problems a weak Harnack inequality with optimal critical exponent. The proof relies on new a priori estimates for time fractional problems and uses Moser's iteration technique and an abstract lemma of Bombieri and Giusti, the latter allowing to avoid the rather technically involved approach via $B M O$. As applications of the weak Harnack inequality we establish the strong maximum principle, the continuity of weak solutions at $t=0$, and a uniqueness theorem for global bounded weak solutions.
\end{abstract}

Mathematics Subject Classification (2010): 35R09 (primary); 45K05 (secondary).

\section{Introduction and main result}

Let $T>0$ and $\Omega$ be a bounded domain in $\mathbb{R}^{N}$. In this paper we are concerned with linear partial integro-differential equations of the form

$$
\partial_{t}^{\alpha}\left(u-u_{0}\right)-\operatorname{div}(A(t, x) D u)=0, \quad t \in(0, T), x \in \Omega .
$$

Here $u_{0}=u_{0}(x)$ is a given initial data for $u, A=\left(a_{i j}\right)$ is $\mathbb{R}^{N \times N}$-valued, $D u$ denotes the spatial gradient of $u$, and $\partial_{t}^{\alpha}$ stands for the Riemann-Liouville fractional derivation operator with respect to time of order $\alpha \in(0,1)$; it is defined by

$$
\partial_{t}^{\alpha} v(t, x)=\partial_{t} \int_{0}^{t} g_{1-\alpha}(t-\tau) v(\tau, x) d \tau, \quad t>0, x \in \Omega,
$$

Work partially supported by the European Community's Human Potential Programme [Evolution Equations for Deterministic and Stochastic Systems], contract code HPRN-CT-2002-00281, and by the Deutsche Forschungsgemeinschaft (DFG), Bonn, Germany.

Received July 23, 2011; accepted December 12, 2011. 
where $g_{\beta}$ denotes the Riemann-Liouville kernel

$$
g_{\beta}(t)=\frac{t^{\beta-1}}{\Gamma(\beta)}, \quad t>0, \beta>0
$$

As to applications, equation (1.1) is a special case of problems arising in mathematical physics when describing dynamic processes in materials with memory, e.g. in the theory of heat conduction with memory, see [24] and the references therein. Time fractional diffusion equations are also used to model anomalous diffusion, see e.g. [20]. In this context, equations of the type (1.1) are termed subdiffusion equations (the time order $\alpha$ lies in $(0,1)$; in the case $\alpha \in(1,2)$, which is not considered here, one speaks of superdiffusion equations. Time fractional diffusion equations of time order $\alpha \in(0,1)$ are closely related to a class of Montroll-Weiss continuous time random walk models where the waiting time density behaves as $t^{-\alpha-1}$ for $t \rightarrow \infty$, see $e . g$. $[14,15,20]$. Problems of the type (1.1) are further used to describe diffusion on fractals $([20,25])$, and they also appear in mathematical finance, see e.g. [27].

Letting $\Omega_{T}=(0, T) \times \Omega$ we will assume that

(H1) $A \in L_{\infty}\left(\Omega_{T} ; \mathbb{R}^{N \times N}\right)$, and

$$
\sum_{i, j=1}^{N}\left|a_{i j}(t, x)\right|^{2} \leq \Lambda^{2}, \quad \text { for a.a. }(t, x) \in \Omega_{T} \text {. }
$$

(H2) There exists $v>0$ such that

$$
(A(t, x) \xi \mid \xi) \geq v|\xi|^{2}, \quad \text { for a.a. }(t, x) \in \Omega_{T}, \text { and all } \xi \in \mathbb{R}^{N}
$$

(H3) $u_{0} \in L_{2}(\Omega)$.

We say that a function $u$ is a weak solution (subsolution, supersolution) of (1.1) in $\Omega_{T}$, if $u$ belongs to the space

$$
\begin{gathered}
Z_{\alpha}:=\left\{v \in L_{\frac{2}{1-\alpha}, w}\left([0, T] ; L_{2}(\Omega)\right) \cap L_{2}\left([0, T] ; H_{2}^{1}(\Omega)\right)\right. \text { such that } \\
\left.g_{1-\alpha} * v \in C\left([0, T] ; L_{2}(\Omega)\right), \text { and }\left.\left(g_{1-\alpha} * v\right)\right|_{t=0}=0\right\},
\end{gathered}
$$

and for any nonnegative test function

$$
\eta \in \stackrel{\circ}{H}_{2}^{1,1}\left(\Omega_{T}\right):=H_{2}^{1}\left([0, T] ; L_{2}(\Omega)\right) \cap L_{2}\left([0, T] ; \stackrel{\circ}{H}_{2}^{1}(\Omega)\right)\left(\stackrel{\circ}{H}_{2}^{1}(\Omega):=\overline{C_{0}^{\infty}(\Omega)} H_{2}^{1}(\Omega)\right)
$$

with $\left.\eta\right|_{t=T}=0$ there holds

$$
\int_{0}^{T} \int_{\Omega}\left(-\eta_{t}\left[g_{1-\alpha} *\left(u-u_{0}\right)\right]+(A D u \mid D \eta)\right) d x d t=(\leq, \geq) 0 .
$$


Here $L_{p, w}$ denotes the weak $L_{p}$ space and $f_{1} * f_{2}$ the convolution on the positive halfline with respect to time, that is $\left(f_{1} * f_{2}\right)(t)=\int_{0}^{t} f_{1}(t-\tau) f_{2}(\tau) d \tau, t \geq 0$.

Weak solutions of (1.1) in the class $Z_{\alpha}$ have been constructed in [37]. Notice also that the function $u_{0}$ plays the role of the initial data for $u$, at least in a weak sense. In case of sufficiently smooth functions $u$ and $g_{1-\alpha} *\left(u-u_{0}\right)$ the condition $\left.\left(g_{1-\alpha} * u\right)\right|_{t=0}=0$ implies $\left.u\right|_{t=0}=u_{0}$, see [37].

To formulate our main result, let $B(x, r)$ denote the open ball with radius $r>0$ centered at $x \in \mathbb{R}^{N}$. By $\mu_{N}$ we mean the Lebesgue measure in $\mathbb{R}^{N}$. For $\delta \in(0,1)$, $t_{0} \geq 0, \tau>0$, and a ball $B\left(x_{0}, r\right)$, define the boxes

$$
\begin{aligned}
& Q_{-}\left(t_{0}, x_{0}, r\right)=\left(t_{0}, t_{0}+\delta \tau r^{2 / \alpha}\right) \times B\left(x_{0}, \delta r\right), \\
& Q_{+}\left(t_{0}, x_{0}, r\right)=\left(t_{0}+(2-\delta) \tau r^{2 / \alpha}, t_{0}+2 \tau r^{2 / \alpha}\right) \times B\left(x_{0}, \delta r\right) .
\end{aligned}
$$

Theorem 1.1. Let $\alpha \in(0,1), T>0$, and $\Omega \subset \mathbb{R}^{N}$ be a bounded domain. Suppose the assumptions $(\mathrm{H} 1)-(\mathrm{H} 3)$ are satisfied. Let further $\delta \in(0,1), \eta>1$, and $\tau>0$ be fixed. Then for any $t_{0} \geq 0$ and $r>0$ with $t_{0}+2 \tau r^{2 / \alpha} \leq T$, any ball $B\left(x_{0}, \eta r\right) \subset \Omega$, any $0<p<\frac{2+N \alpha}{2+N \alpha-2 \alpha}$, and any nonnegative weak supersolution $u$ of (1.1) in $\left(0, t_{0}+2 \tau r^{2 / \alpha}\right) \times B\left(x_{0}, \eta r\right)$ with $u_{0} \geq 0$ in $B\left(x_{0}, \eta r\right)$, there holds

$$
\left(\frac{1}{\mu_{N+1}\left(Q_{-}\left(t_{0}, x_{0}, r\right)\right)} \int_{Q_{-}\left(t_{0}, x_{0}, r\right)} u^{p} d \mu_{N+1}\right)^{1 / p} \leq C \underset{Q_{+}\left(t_{0}, x_{0}, r\right)}{\operatorname{essinf}} u,
$$

where the constant $C=C(\nu, \Lambda, \delta, \tau, \eta, \alpha, N, p)$.

Theorem 1.1 states that nonnegative weak supersolutions of (1.1) satisfy a weak form of Harnack inequality in the sense that we do not have an estimate for the supremum of $u$ on $Q_{-}\left(t_{0}, x_{0}, r\right)$ but only an $L_{p}$ estimate. We also show that the critical exponent $\frac{2+N \alpha}{2+N \alpha-2 \alpha}$ is optimal, i.e. the inequality fails to hold for $p \geq \frac{2+N \alpha}{2+N \alpha-2 \alpha}$.

Theorem 1.1 can be viewed as the time fractional analogue of the corresponding result in the classical parabolic case $\alpha=1$, see e.g. [19, Theorem 6.18] and [29]. Sending $\alpha \rightarrow 1$ in the expression for the critical exponent yields $1+2 / N$, which is the well-known critical exponent for the heat equation. We would like to point out that the statement of Theorem 1.1 remains valid for (appropriately defined) weak supersolutions of (1.1) on $\left(t_{0}, t_{0}+2 \tau r^{2 / \alpha}\right) \times B\left(x_{0}, \eta r\right)$ which are nonnegative on $\left(0, t_{0}+2 \tau r^{2 / \alpha}\right) \times B\left(x_{0}, \eta r\right)$. To avoid further technicalities we have confined ourselves to consider only weak supersolutions on the full time-interval $\left(0, t_{0}+2 \tau r^{2 / \alpha}\right)$. Note that the global positivity assumption cannot be replaced by a local one, as simple examples show, $c f$. [36]. This significant difference to the case $\alpha=1$ is due to the non-local nature of $\partial_{t}^{\alpha}$. The same phenomenon is known for integro-differential operators like $(-\Delta)^{\alpha}$ with $\alpha \in(0,1)$, see e.g. [16].

As a simple consequence of the weak Harnack inequality we derive the strong maximum principle for weak subsolutions of (1.1), see Theorem 5.1 below. The weak maximum principle has been proven in [33], even in a more general setting. 
As a further consequence of the weak Harnack inequality we obtain a uniqueness theorem for global bounded weak solutions, see Corollary 5.3 below. It states that any bounded weak solution of (1.1) on $\mathbb{R}_{+} \times \mathbb{R}^{N}$ with $u_{0}=0$ vanishes a.e. on $\mathbb{R}_{+} \times \mathbb{R}^{N}$.

The proof of Theorem 1.1 relies on new a priori estimates for time fractional problems, which are derived by means of the fundamental identity (2.6) (see below) for the regularized fractional derivative. It further uses Moser's iteration technique and an elementary but subtle lemma of Bombieri and Giusti [2], which allows to avoid the rather technically involved approach via $B M O$-functions. This simplification is already of great significance in the classical parabolic case, see Moser [23] and Saloff-Coste [26].

One of the technical difficulties in deriving the desired estimates in the weak setting is to find an appropriate time regularization of the problem. In the case $\alpha=1$ this can be achieved by means of Steklov averages in time. In the time fractional case this method does not work anymore, since Steklov average operators and convolution do not commute. It turns out that instead one can use the Yosida approximation of the fractional derivative, which leads to a regularization of the kernel $g_{1-\alpha}$. This method has already been used in [12,30,37], and [33].

We point out that the results obtained in this paper can be generalized to quasilinear equations of the form

$$
\partial_{t}^{\alpha}\left(u-u_{0}\right)-\operatorname{div} a(t, x, u, D u)=b(t, x, u, D u), t \in(0, T), x \in \Omega,
$$

with suitable structure conditions on the functions $a$ and $b$. This is possible, as also known from the elliptic and the classical parabolic case, since the test function method used in the proof of Theorem 1.1 does not depend so much on the linearity of the differential operator w.r.t. the spatial variables but on a certain nonlinear structure, $c f$. [11, 19,29], and [33].

In the literature there exist many papers where equations of the type (1.1), as well as nonlinear or abstract variants of them are studied in a strong setting, assuming more smoothness on the coefficients and nonlinearities, see e.g. $[1,4,7$, $10,12,24,34,35]$. Concerning the weak setting described above one finds only a few results. Existence of weak solutions has been shown in [37] in an abstract setting for a more general class of kernels. Boundedness of weak solutions has been obtained in [33] in the quasilinear case by means of the De Giorgi technique. In the very recent work [31], it is proved that any weak solution of (1.1) is Hölder continuous in the interior of the parabolic domain. The proof is quite involved and relies on the De Giorgi technique and the method of non-local growth lemmata ( $c f$. [28]). With the weak Harnack inequality, the present paper establishes another key result towards a De Giorgi-Nash-Moser theory for time fractional evolution equations in divergence form of order $\alpha \in(0,1)$.

In the classical parabolic case boundedness and the weak (or full) Harnack inequality imply an Hölder estimate for weak solutions, $c f$. [9, 18, 19, 22]. We also refer to [11] and [21] for the elliptic case. In the present situation one cannot argue anymore as in the classical parabolic case, due to the global positivity assumption 
in Theorem 1.1. The same problem arises for the fractional Laplacian, see [28]. However, in our case it is possible to establish without much effort at least continuity at $t=0$. This is done in Theorem 5.2 in the case $u_{0}=0$. It is shown that in this case any bounded weak solution $u$ of (1.1) is continuous at $\left(0, x_{0}\right)$ for all $x_{0} \in \Omega$ and $\lim _{(t, x) \rightarrow\left(0, x_{0}\right)} u(t, x)=0$. Thus for such weak solutions the initial condition $\left.u\right|_{t=0}=0$ is satisfied in the classical sense.

We further remark that in the purely time-dependent case, that is for scalar equations of the form

$$
\partial_{t}^{\alpha}\left(u-u_{0}\right)+\sigma u=0, \quad t \in(0, T),
$$

with $\sigma \geq 0$, a weak Harnack inequality with optimal exponent $1 /(1-\alpha)$ has been proven in [32] for nonnegative supersolutions. Recently, the full Harnack inequality for nonnegative solutions has been established in [36]. This, together with the above results, indicates that the full Harnack inequality should also hold for nonnegative solutions of (1.1), which is still an open problem, even in the case $A(t, x) \equiv I d$.

The paper is organized as follows. In Section 2 we collect the basic tools needed for the proof of Theorem 1.1. These include two abstract lemmas on Moser iterations and the lemma of Bombieri and Giusti. We further explain the approximation method for the fractional derivation operator and state the fundamental identity (2.6), which is frequently used in Section 3, where we give the proof of the main result. In Section 4 we show that the critical exponent in Theorem 1.1 is optimal. Finally, Section 5 is devoted to applications of the weak Harnack inequality.

ACKNOWLEDGEMENTS. This paper was initiated while the author was visiting the Technical University Delft (NL) in 2003/2004. The author is greatly indebted to Philippe Clément for many fruitful discussions and valuable suggestions.

\section{Preliminaries}

\subsection{Moser iterations and an abstract lemma of Bombieri and Giusti}

Throughout this subsection $U_{\sigma}, 0<\sigma \leq 1$, will denote a collection of measurable subsets of a fixed finite measure space endowed with a measure $\mu$, such that $U_{\sigma^{\prime}} \subset$ $U_{\sigma}$ if $\sigma^{\prime} \leq \sigma$. For $p \in(0, \infty)$ and $0<\sigma \leq 1, L_{p}\left(U_{\sigma}\right)$ stands for the Lebesgue space $L_{p}\left(U_{\sigma}, d \mu\right)$ of all $\mu$-measurable functions $f: U_{\sigma} \rightarrow \mathbb{R}$ with $|f|_{L_{p}\left(U_{\sigma}\right)}:=$ $\left(\int_{U_{\sigma}}|f|^{p} d \mu\right)^{1 / p}<\infty$.

The following two lemmas are basic to Moser's iteration technique. The arguments in their proofs have been repeatedly used in the literature (see e.g. $[11,19,21$, $22,26,29]$ ), so it is worthwhile to formulate them as lemmas in abstract form, also for future reference. We provide proofs for the sake of completeness.

The first Moser iteration result reads as follows, see also [8, Lemma 2.3]. 
Lemma 2.1. Let $\kappa>1, \bar{p} \geq 1, C \geq 1$, and $\gamma>0$. Suppose $f$ is a $\mu$-measurable function on $U_{1}$ such that

$$
|f|_{L_{\beta \kappa}\left(U_{\sigma^{\prime}}\right)} \leq\left(\frac{C(1+\beta)^{\gamma}}{\left(\sigma-\sigma^{\prime}\right)^{\gamma}}\right)^{1 / \beta}|f|_{L_{\beta}\left(U_{\sigma}\right)}, \quad 0<\sigma^{\prime}<\sigma \leq 1, \beta>0 .
$$

Then there exist constants $M=M(C, \gamma, \kappa, \bar{p})$ and $\gamma_{0}=\gamma_{0}(\gamma, \kappa)$ such that

$$
\underset{U_{\delta}}{\operatorname{ess} \sup }|f| \leq\left(\frac{M}{(1-\delta)^{\gamma_{0}}}\right)^{1 / p}|f|_{L_{p}\left(U_{1}\right)} \text { for all } \delta \in(0,1), p \in(0, \bar{p}] \text {. }
$$

Proof. For $q>0$ and $0<\sigma \leq 1$, let

$$
\Phi(q, \sigma)=\left(\int_{U_{\sigma}}|f|^{q} d \mu\right)^{1 / q}
$$

Let $0<p \leq \bar{p}$ and $\delta \in(0,1)$. Set $p_{i}=p \kappa^{i}, i=0,1, \ldots$ and define the sequence $\left\{\sigma_{i}\right\}, i=0,1, \ldots$, by $\sigma_{0}=1$ and $\sigma_{i}=1-\sum_{j=1}^{i} 2^{-j}(1-\delta), i=1,2, \ldots$; observe that $1=\sigma_{0}>\sigma_{1}>\ldots>\sigma_{i}>\sigma_{i+1}>\delta$ as well as $\sigma_{i-1}-\sigma_{i}=2^{-i}(1-\delta), i \geq 1$. Suppose now $n \in \mathbb{N}$. By using (2.1) with $\beta=p_{i}, i=0,1, \ldots, n-1$, we obtain

$$
\begin{aligned}
\Phi\left(p_{n}, \delta\right) & \leq \Phi\left(p_{n}, \sigma_{n}\right)=\Phi\left(p_{n-1} \kappa, \sigma_{n}\right) \\
& \leq\left(\frac{C\left(1+p \kappa^{n-1}\right)^{\gamma}}{\left[2^{-n}(1-\delta)\right]^{\gamma}}\right)^{\frac{1}{p} \kappa^{-(n-1)}} \Phi\left(p_{n-1}, \sigma_{n-1}\right) \\
& \leq\left(\frac{C\left(2 \bar{p} \kappa^{n-1}\right)^{\gamma}}{\left[2^{-n}(1-\delta)\right]^{\gamma}}\right)^{\frac{1}{p} \kappa^{-(n-1)}} \Phi\left(p_{n-1}, \sigma_{n-1}\right) \\
& \leq\left(\frac{\tilde{C}(C, \bar{p}, \gamma)^{n} \kappa^{\gamma(n-1)}}{(1-\delta)^{\gamma}}\right)^{\frac{1}{p} \kappa^{-(n-1)}} \Phi\left(p_{n-1}, \sigma_{n-1}\right) \leq \ldots \\
& \leq\left(\tilde{C}_{j=0}^{\sum_{j-1}^{n-1}(j+1) \kappa^{-j}} \kappa^{\gamma \sum_{j=0}^{n-1} j \kappa^{-j}}(1-\delta)^{-\gamma \sum_{j=0}^{n-1} \kappa^{-j}}\right)^{1 / p} \Phi\left(p_{0}, \sigma_{0}\right) \\
& \leq\left(\frac{M(C, \bar{p}, \gamma, \kappa)}{(1-\delta)^{\frac{\gamma \kappa}{\kappa-1}}}\right)^{1 / p} \Phi(p, 1) .
\end{aligned}
$$

We let now $n$ tend to $\infty$ and use the fact that

$$
\lim _{n \rightarrow \infty} \Phi\left(p_{n}, \delta\right)=\underset{U_{\delta}}{\operatorname{ess} \sup }|f|
$$

to get

$$
\underset{U_{\delta}}{\operatorname{ess} \sup }|f| \leq\left(\frac{M(C, \bar{p}, \gamma, \kappa)}{(1-\delta)^{\frac{\gamma \kappa}{\kappa-1}}}\right)^{1 / p}|f|_{L_{p}\left(U_{1}\right)}
$$

Hence the proof is complete.

The second Moser iteration result is the following, see also [8, Lemma 2.5]. 
Lemma 2.2. Assume that $\mu\left(U_{1}\right) \leq 1$. Let $\kappa>1,0<p_{0}<\kappa$, and $C \geq 1, \gamma>0$. Suppose $f$ is a $\mu$-measurable function on $U_{1}$ such that

$$
\begin{aligned}
& |f|_{L_{\beta \kappa}\left(U_{\sigma^{\prime}}\right)} \leq\left(\frac{C}{\left(\sigma-\sigma^{\prime}\right)^{\gamma}}\right)^{1 / \beta}|f|_{L_{\beta}\left(U_{\sigma}\right)}, \\
& 0<\sigma^{\prime}<\sigma \leq 1,0<\beta \leq \frac{p_{0}}{\kappa}<1 .
\end{aligned}
$$

Then there exist constants $M=M(C, \gamma, \kappa)$ and $\gamma_{0}=\gamma_{0}(\gamma, \kappa)$ such that

$$
|f|_{L_{p_{0}}\left(U_{\delta}\right)} \leq\left(\frac{M}{(1-\delta)^{\gamma_{0}}}\right)^{1 / p-1 / p_{0}}|f|_{L_{p}\left(U_{1}\right)} \quad \text { for all } \delta \in(0,1), p \in\left(0, \frac{p_{0}}{\kappa}\right] \text {. }
$$

Proof. Set $p_{i}=p_{0} \kappa^{-i}, i=1,2, \ldots$ Given $\delta \in(0,1)$ we take again the sequence $\left\{\sigma_{i}\right\}, i=0,1,2, \ldots$, defined by $\sigma_{0}=1$ and $\sigma_{i}=1-\sum_{j=1}^{i} 2^{-j}(1-\delta), i \geq 1$. Suppose now $n \in \mathbb{N}$. By using (2.2) with $\beta=p_{i}, i=1, \ldots, n$, we obtain

$$
\begin{aligned}
\Phi\left(p_{0}, \delta\right) & \leq \Phi\left(p_{0}, \sigma_{n}\right)=\Phi\left(p_{1} \kappa, \sigma_{n}\right) \leq \frac{C^{\kappa / p_{0}}}{\left[2^{-n}(1-\delta)\right]^{\gamma \kappa / p_{0}}} \Phi\left(p_{1}, \sigma_{n-1}\right) \\
& \leq \frac{C^{\kappa / p_{0}}}{\left[2^{-n}(1-\delta)\right]^{\gamma \kappa / p_{0}}} \frac{C^{\kappa^{2} / p_{0}}}{\left[2^{-(n-1)}(1-\delta)\right]^{\gamma \kappa^{2} / p_{0}}} \Phi\left(p_{2}, \sigma_{n-2}\right) \leq \ldots \\
& \leq \frac{C^{\frac{1}{p_{0}}\left(\kappa+\kappa^{2}+\ldots+\kappa^{n}\right)}}{2^{-\frac{\gamma}{p_{0}}\left(n \kappa+(n-1) \kappa^{2}+\ldots+2 \kappa^{n-1}+\kappa^{n}\right)}(1-\delta)^{\frac{\gamma}{p_{0}}\left(\kappa+\kappa^{2}+\ldots+\kappa^{n}\right)}} \Phi\left(p_{n}, \sigma_{0}\right) .
\end{aligned}
$$

Since $p_{i}=p_{0} \kappa^{-i}$, we have

$$
\frac{1}{p_{0}} \sum_{j=1}^{n} \kappa^{j}=\frac{\kappa\left(\kappa^{n}-1\right)}{p_{0}(\kappa-1)}=\frac{\kappa}{p_{0}(\kappa-1)}\left(\frac{p_{0}}{p_{n}}-1\right)=\frac{\kappa}{\kappa-1}\left(\frac{1}{p_{n}}-\frac{1}{p_{0}}\right) .
$$

Employing the formula

$$
\sum_{j=1}^{n} j \kappa^{j-1}=\frac{1-(n+1) \kappa^{n}+n \kappa^{n+1}}{(\kappa-1)^{2}}
$$

we have further

$$
\begin{aligned}
\sum_{j=1}^{n}(n+1-j) \kappa^{j} & =(n+1) \sum_{j=1}^{n} \kappa^{j}-\sum_{j=1}^{n} j \kappa^{j} \\
& =(n+1) \kappa \frac{\kappa^{n}-1}{\kappa-1}-\kappa \frac{1-(n+1) \kappa^{n}+n \kappa^{n+1}}{(\kappa-1)^{2}} \\
& =\kappa \frac{\kappa^{n+1}-(n+1) \kappa+n}{(\kappa-1)^{2}} \leq \frac{\kappa}{(\kappa-1)^{2}} \kappa^{n+1} \\
& \leq \frac{\kappa^{3}}{(\kappa-1)^{3}}\left(\kappa^{n}-1\right) \leq \frac{\kappa^{3}}{(\kappa-1)^{3}}\left(\frac{p_{0}}{p_{n}}-1\right),
\end{aligned}
$$


which yields

$$
\frac{1}{p_{0}} \sum_{j=1}^{n}(n+1-j) \kappa^{j} \leq \frac{\kappa^{3}}{(\kappa-1)^{3}}\left(\frac{1}{p_{n}}-\frac{1}{p_{0}}\right)
$$

Therefore

$$
\Phi\left(p_{0}, \delta\right) \leq\left[\frac{2^{\frac{\gamma \kappa^{3}}{(\kappa-1)^{3}}} C^{\frac{\kappa}{\kappa-1}}}{(1-\delta)^{\frac{\gamma \kappa}{\kappa-1}}}\right]^{\frac{1}{p_{n}}-\frac{1}{p_{0}}} \Phi\left(p_{n}, \sigma_{0}\right) .
$$

Given $p \in\left(0, p_{0} / \kappa\right]$ there exists $n \geq 2$ such that $p_{n}<p \leq p_{n-1}$. We then have

$$
\begin{aligned}
\frac{1}{p_{n}}-\frac{1}{p_{0}} & =\frac{\kappa^{n}-1}{p_{0}} \leq \frac{\kappa^{n}+\kappa^{n-1}-\kappa-1}{p_{0}}=\frac{(1+\kappa)\left(\kappa^{n-1}-1\right)}{p_{0}} \\
& =(1+\kappa)\left(\frac{1}{p_{n-1}}-\frac{1}{p_{0}}\right) \leq(1+\kappa)\left(\frac{1}{p}-\frac{1}{p_{0}}\right)
\end{aligned}
$$

as well as

$$
\Phi\left(p_{n}, \sigma_{0}\right)=\Phi\left(p_{n}, 1\right) \leq \Phi(p, 1),
$$

by Hölder's inequality and the assumption $\mu\left(U_{1}\right) \leq 1$. All in all, we obtain

$$
\Phi\left(p_{0}, \delta\right) \leq\left[\frac{2^{\frac{\gamma \kappa^{3}}{(\kappa-1)^{3}}} C^{\frac{\kappa}{\kappa-1}}}{(1-\delta)^{\frac{\gamma \kappa}{\kappa-1}}}\right]^{(1+\kappa)\left(\frac{1}{p}-\frac{1}{p_{0}}\right)} \Phi(p, 1)
$$

which proves the lemma.

The following abstract lemma is due to Bombieri and Giusti [2]. For a proof we also refer to [26, Lemma 2.2.6] and [8, Lemma 2.6].

Lemma 2.3. Let $\delta, \eta \in(0,1)$, and let $\gamma, C$ be positive constants and $0<\beta_{0} \leq \infty$. Suppose $f$ is a positive $\mu$-measurable function on $U_{1}$ which satisfies the following two conditions:

$$
|f|_{L_{\beta_{0}}\left(U_{\sigma^{\prime}}\right)} \leq\left[C\left(\sigma-\sigma^{\prime}\right)^{-\gamma} \mu\left(U_{1}\right)^{-1}\right]^{1 / \beta-1 / \beta_{0}}|f|_{L_{\beta}\left(U_{\sigma}\right)},
$$

for all $\sigma, \sigma^{\prime}, \beta$ such that $0<\delta \leq \sigma^{\prime}<\sigma \leq 1$ and $0<\beta \leq \min \left\{1, \eta \beta_{0}\right\}$.

$$
\mu(\{\log f>\lambda\}) \leq C \mu\left(U_{1}\right) \lambda^{-1}
$$

for all $\lambda>0$.

Then

$$
|f|_{L_{\beta_{0}}\left(U_{\delta}\right)} \leq M \mu\left(U_{1}\right)^{1 / \beta_{0}},
$$

where $M$ depends only on $\delta, \eta, \gamma, C$, and $\beta_{0}$. 


\subsection{The Yosida approximation of the fractional derivation operator}

Let $0<\alpha<1,1 \leq p<\infty, T>0$, and $X$ be a real Banach space. Then the fractional derivation operator $B$ defined by

$B u=\frac{d}{d t}\left(g_{1-\alpha} * u\right), \quad D(B)=\left\{u \in L_{p}([0, T] ; X): g_{1-\alpha} * u \in{ }_{0} H_{p}^{1}([0, T] ; X)\right\}$,

where the zero means vanishing at $t=0$, is known to be $m$-accretive in $L_{p}([0, T] ; X)$, $c f$. [3,6], and [12]. Its Yosida approximations $B_{n}$, defined by $B_{n}=n B(n+$ $B)^{-1}, n \in \mathbb{N}$, enjoy the property that for any $u \in D(B)$, one has $B_{n} u \rightarrow B u$ in $L_{p}([0, T] ; X)$ as $n \rightarrow \infty$. Further, one has the representation

$$
B_{n} u=\frac{d}{d t}\left(g_{1-\alpha, n} * u\right), \quad u \in L_{p}([0, T] ; X), n \in \mathbb{N},
$$

where $g_{1-\alpha, n}=n s_{\alpha, n}$, and $s_{\alpha, n}$ is the unique solution of the scalar-valued Volterra equation

$$
s_{\alpha, n}(t)+n\left(s_{\alpha, n} * g_{\alpha}\right)(t)=1, \quad t>0, n \in \mathbb{N},
$$

see $e . g$. [30]. Let $h_{\alpha, n} \in L_{1, \text { loc }}\left(\mathbb{R}_{+}\right)$be the resolvent kernel associated with $n g_{\alpha}$, that is

$$
h_{\alpha, n}(t)+n\left(h_{\alpha, n} * g_{\alpha}\right)(t)=n g_{\alpha}(t), \quad t>0, n \in \mathbb{N} .
$$

Convolving (2.4) with $g_{1-\alpha}$ and using $g_{\alpha} * g_{1-\alpha}=1$, we obtain

$$
\left(g_{1-\alpha} * h_{\alpha, n}\right)(t)+n\left(\left[g_{1-\alpha} * h_{\alpha, n}\right] * g_{\alpha}\right)(t)=n, \quad t>0, n \in \mathbb{N} .
$$

Hence

$$
g_{1-\alpha, n}=n s_{\alpha, n}=g_{1-\alpha} * h_{\alpha, n}, \quad n \in \mathbb{N} .
$$

The kernels $g_{1-\alpha, n}$ are nonnegative and nonincreasing for all $n \in \mathbb{N}$, and they belong to $H_{1}^{1}([0, T]), c f$. [24] and [30]. Note that for any function $f \in L_{p}([0, T] ; X)$, $1 \leq p<\infty$, there holds $h_{\alpha, n} * f \rightarrow f$ in $L_{p}([0, T] ; X)$ as $n \rightarrow \infty$. In fact, setting $u=g_{\alpha} * f$, we have $u \in D(B)$, and

$$
\begin{aligned}
B_{n} u & =\frac{d}{d t}\left(g_{1-\alpha, n} * u\right)=\frac{d}{d t}\left(g_{1-\alpha} * g_{\alpha} * h_{\alpha, n} * f\right)=h_{\alpha, n} * f \rightarrow B u \\
& =f \quad \text { in } L_{p}([0, T] ; X)
\end{aligned}
$$

as $n \rightarrow \infty$. In particular, $g_{1-\alpha, n} \rightarrow g_{1-\alpha}$ in $L_{1}([0, T])$ as $n \rightarrow \infty$.

We next state a fundamental identity for integro-differential operators of the form $\frac{d}{d t}(k * u), c f$. also [33]. Suppose $k \in H_{1}^{1}([0, T])$ and $H \in C^{1}(\mathbb{R})$. Then it follows from a straightforward computation that for any sufficiently smooth function $u$ on $(0, T)$ one has for a.a. $t \in(0, T)$,

$$
\begin{aligned}
& H^{\prime}(u(t)) \frac{d}{d t}(k * u)(t)=\frac{d}{d t}(k * H(u))(t)+\left(-H(u(t))+H^{\prime}(u(t)) u(t)\right) k(t) \\
& +\int_{0}^{t}\left(H(u(t-s))-H(u(t))-H^{\prime}(u(t))[u(t-s)-u(t)]\right)[-\dot{k}(s)] d s,
\end{aligned}
$$


where $k$ denotes the derivative of $k$. In particular this identity applies to the Yosida approximations of the fractional derivation operator. We remark that an integrated version of (2.6) can be found in [13, Lemma 18.4.1].

Observe that the last term in (2.6) is nonnegative in case $H$ is convex and $k$ is nonincreasing. This fact is frequently used in the estimates below. However we would like to point out that for the delicate logarithmic estimates in Section 3.3 one really needs the full identity (2.6), even though we work all the time with convex or concave functions. In particular, the crucial fractional differential inequality (3.40) cannot be obtained by using merely convexity inequalities.

The subsequent two lemmas are also obtained by simple algebra.

Lemma 2.4. Let $T>0$ and $\alpha \in(0,1)$. Suppose that $v \in{ }_{0} H_{1}^{1}([0, T])$ and $\varphi \in$ $C^{1}([0, T])$. Then

$$
\begin{array}{r}
\left(g_{\alpha} *(\varphi \dot{v})\right)(t)=\varphi(t)\left(g_{\alpha} * \dot{v}\right)(t)+\int_{0}^{t} v(\sigma) \partial_{\sigma}\left(g_{\alpha}(t-\sigma)[\varphi(t)-\varphi(\sigma)]\right) d \sigma, \\
\text { a.a. } t \in(0, T) .
\end{array}
$$

If in addition $v$ is nonnegative and $\varphi$ is nondecreasing there holds

$$
\left(g_{\alpha} *(\varphi \dot{v})\right)(t) \geq \varphi(t)\left(g_{\alpha} * \dot{v}\right)(t)-\int_{0}^{t} g_{\alpha}(t-\sigma) \dot{\varphi}(\sigma) v(\sigma) d \sigma, \text { a.a. } t \in(0, T) .
$$

Lemma 2.5. Let $T>0, k \in H_{1}^{1}([0, T]), v \in L_{1}([0, T])$, and $\varphi \in C^{1}([0, T])$. Then $\varphi(t) \frac{d}{d t}(k * v)(t)=\frac{d}{d t}(k *[\varphi v])(t)+\int_{0}^{t} \dot{k}(t-\tau)(\varphi(t)-\varphi(\tau)) v(\tau) d \tau$, a.a. $t \in(0, T)$.

\subsection{An embedding result and a weighted Poincaré inequality}

Let $T>0$ and $\Omega$ be a bounded domain in $\mathbb{R}^{N}$. For $1<p \leq \infty$ we define the space

$$
V_{p}:=V_{p}([0, T] \times \Omega)=L_{2 p}\left([0, T] ; L_{2}(\Omega)\right) \cap L_{2}\left([0, T] ; H_{2}^{1}(\Omega)\right),
$$

endowed with the norm

$$
|u|_{V_{p}([0, T] \times \Omega)}:=|u|_{L_{2 p}\left([0, T] ; L_{2}(\Omega)\right)}+|D u|_{L_{2}\left([0, T] ; L_{2}(\Omega)\right)} .
$$

Set

$$
\kappa:=\kappa_{p}:=\frac{2 p+N(p-1)}{2+N(p-1)}
$$

with $\kappa_{\infty}=1+2 / N$. Then $V_{p} \hookrightarrow L_{2 \kappa}([0, T] \times \Omega)$, and

$$
|u|_{L_{2 \kappa}([0, T] \times \Omega)} \leq C(N, p)|u|_{V_{p}([0, T] \times \Omega)},
$$


for all $u \in V_{p} \cap L_{2}\left([0, T] ; \stackrel{H}{2}_{2}^{1}(\Omega)\right)$. This is a consequence of the GagliardoNirenberg and Hölder's inequality. The case $p=\infty$ is contained, $e . g$., in [18, page 74 and 75]. The proof given there easily extends to the general case. For a more general embedding result (without proof) we also refer to [33, Section 2].

The following result can be found in [22, Lemma 3], see also [19, Lemma 6.12].

Proposition 2.6. Let $\varphi \in C\left(\mathbb{R}^{N}\right)$ with non-empty compact support of diameter $d$ and assume that $0 \leq \varphi \leq 1$. Suppose that the domains $\left\{x \in \mathbb{R}^{N}: \varphi(x) \geq a\right\}$ are convex for all $a \leq 1$. Then for any function $u \in H_{2}^{1}\left(\mathbb{R}^{N}\right)$,

$$
\int_{\mathbb{R}^{N}}\left(u(x)-u_{\varphi}\right)^{2} \varphi(x) d x \leq \frac{2 d^{2} \mu_{N}(\operatorname{supp} \varphi)}{|\varphi|_{L_{1}\left(\mathbb{R}^{N}\right)}} \int_{\mathbb{R}^{N}}|D u(x)|^{2} \varphi(x) d x,
$$

where

$$
u_{\varphi}=\frac{\int_{\mathbb{R}^{N}} u(x) \varphi(x) d x}{\int_{\mathbb{R}^{N}} \varphi(x) d x} .
$$

\section{Proof of the main result}

\subsection{The regularized weak formulation, time shifts, and scalings}

The following lemma is basic to deriving a priori estimates for weak (sub-/super-) solutions of (1.1). It provides an equivalent weak formulation of (1.1) where the singular kernel $g_{1-\alpha}$ is replaced by the more regular kernel $g_{1-\alpha, n}(n \in \mathbb{N})$ given in (2.5). In what follows the kernels $h_{n}:=h_{\alpha, n}, n \in \mathbb{N}$, are defined as in Section 2.2.

Lemma 3.1. Let $\alpha \in(0,1), T>0$, and $\Omega \subset \mathbb{R}^{N}$ be a bounded domain. Suppose the assumptions (H1)-(H3) are satisfied. Then $u \in Z_{\alpha}$ is a weak solution (subsolution, supersolution) of (1.1) in $\Omega_{T}$ if and only if for any nonnegative function $\psi \in \stackrel{\circ}{H}_{2}^{1}(\Omega)$ one has

$$
\begin{array}{r}
\int_{\Omega}\left(\psi \partial_{t}\left[g_{1-\alpha, n} *\left(u-u_{0}\right)\right]+\left(h_{n} *[A D u] \mid D \psi\right)\right) d x=(\leq, \geq) 0, \\
\text { a.a.t } \in(0, T), n \in \mathbb{N} .
\end{array}
$$

For a proof we refer to [33, Lemma 3.1], where a more general situation is considered with a slightly different function space for the solution. The proof of Lemma 3.1 is analogous.

Let $u \in Z_{\alpha}$ be a weak supersolution of (1.1) in $\Omega_{T}$ and assume that $u_{0} \geq 0$ in $\Omega$. Then Lemma 3.1 and positivity of $g_{1-\alpha, n}$ imply that

$$
\int_{\Omega}\left(\psi \partial_{t}\left(g_{1-\alpha, n} * u\right)+\left(h_{n} *[A D u] \mid D \psi\right)\right) d x \geq 0, \quad \text { a.a. } t \in(0, T), n \in \mathbb{N},
$$

for any nonnegative function $\psi \in \hat{H}_{2}^{1}(\Omega)$. 
Let now $t_{1} \in(0, T)$ be fixed. For $t \in\left(t_{1}, T\right)$ we introduce the shifted time $s=t-t_{1}$ and set $\tilde{f}(s)=f\left(s+t_{1}\right), s \in\left(0, T-t_{1}\right)$, for functions $f$ defined on $\left(t_{1}, T\right)$. From the decomposition

$$
\begin{array}{r}
\left(g_{1-\alpha, n} * u\right)(t, x)=\int_{t_{1}}^{t} g_{1-\alpha, n}(t-\tau) u(\tau, x) d \tau+\int_{0}^{t_{1}} g_{1-\alpha, n}(t-\tau) u(\tau, x) d \tau, \\
t \in\left(t_{1}, T\right),
\end{array}
$$

we then deduce that

$$
\partial_{t}\left(g_{1-\alpha, n} * u\right)(t, x)=\partial_{s}\left(g_{1-\alpha, n} * \tilde{u}\right)(s, x)+\int_{0}^{t_{1}} \dot{g}_{1-\alpha, n}\left(s+t_{1}-\tau\right) u(\tau, x) d \tau .
$$

Assuming in addition that $u \geq 0$ on $\left(0, t_{1}\right) \times \Omega$ it follows from (3.1), (3.2), and the positivity of $\psi$ and of $-\dot{g}_{1-\alpha, n}$ that

$$
\begin{array}{r}
\int_{\Omega}\left(\psi \partial_{s}\left(g_{1-\alpha, n} * \tilde{u}\right)+\left(\left(h_{n} *[A D u]\right)^{\sim} \mid D \psi\right)\right) d x \geq 0, \\
\text { a.a. } s \in\left(0, T-t_{1}\right), n \in \mathbb{N},
\end{array}
$$

for any nonnegative function $\psi \in \stackrel{\circ}{H}_{2}^{1}(\Omega)$. This relation will be the starting point for all of the estimates below.

We conclude this section with a remark on the scaling properties of equation (1.1). Let $t_{0}, r>0$ and $x_{0} \in \mathbb{R}^{N}$. Suppose $u \in Z_{\alpha}$ is a weak solution (subsolution, supersolution) of $(1.1)$ in $\left(0, t_{0} r^{2 / \alpha}\right) \times B\left(x_{0}, r\right)$. Changing the coordinates according to $s=t / r^{2 / \alpha}$ and $y=\left(x-x_{0}\right) / r$ and setting $v(s, y)=u\left(s r^{2 / \alpha}, x_{0}+y r\right), v_{0}(y)=$ $u_{0}\left(x_{0}+y r\right)$, and $\tilde{A}(s, y)=A\left(s r^{2 / \alpha}, x_{0}+y r\right)$, the problem for $u$ is transformed to a problem for $v$ in $\left(0, t_{0}\right) \times B(0,1)$, namely there holds with $D=D_{y}$ (also in the weak sense)

$$
\partial_{s}^{\alpha}\left(v-v_{0}\right)-\operatorname{div}(\tilde{A}(s, y) D v)=(\leq, \geq) 0, \quad s \in\left(0, t_{0}\right), y \in B(0,1) .
$$

\subsection{Mean value inequalities}

For $\sigma>0$ we put $\sigma B(x, r):=B(x, \sigma r)$. Recall that $\mu_{N}$ denotes the Lebesgue measure in $\mathbb{R}^{N}$.

Theorem 3.2. Let $\alpha \in(0,1), T>0$, and $\Omega \subset \mathbb{R}^{N}$ be a bounded domain. Suppose the assumptions (H1)-(H3) are satisfied. Let $\eta>0$ and $\delta \in(0,1)$ be fixed. Then for any $t_{0} \in(0, T]$ and $r>0$ with $t_{0}-\eta r^{2 / \alpha} \geq 0$, any ball $B=B\left(x_{0}, r\right) \subset \Omega$, and any weak supersolution $u \geq \varepsilon>0$ of $(1.1)$ in $\left(0, t_{0}\right) \times B$ with $u_{0} \geq 0$ in $B$, there holds

$$
\underset{U_{\sigma^{\prime}}}{\operatorname{ess} \sup } u^{-1} \leq\left(\frac{C \mu_{N+1}\left(U_{1}\right)^{-1}}{\left(\sigma-\sigma^{\prime}\right)^{\tau_{0}}}\right)^{1 / \gamma}\left|u^{-1}\right|_{L_{\gamma}\left(U_{\sigma}\right)}, \quad \delta \leq \sigma^{\prime}<\sigma \leq 1, \gamma \in(0,1] \text {. }
$$

Here $U_{\sigma}=\left(t_{0}-\sigma \eta r^{2 / \alpha}, t_{0}\right) \times \sigma B, 0<\sigma \leq 1, C=C(\nu, \Lambda, \delta, \eta, \alpha, N)$ and $\tau_{0}=\tau_{0}(\alpha, N)$. 
Proof. We may assume that $r=1$ and $x_{0}=0$. In fact, in the general case we change coordinates as $t \rightarrow t / r^{2 / \alpha}$ and $x \rightarrow\left(x-x_{0}\right) / r$, thereby transforming the equation to a problem of the same type on $\left(0, t_{0} / r^{2 / \alpha}\right) \times B(0,1), c f$. Section 3.1.

Fix $\sigma^{\prime}$ and $\sigma$ such that $\delta \leq \sigma^{\prime}<\sigma \leq 1$ and put $B_{1}=\sigma B$. For $\rho \in(0,1]$ we set $V_{\rho}=U_{\rho \sigma}$. Given $0<\rho^{\prime}<\rho \leq 1$, let $t_{1}=t_{0}-\rho \sigma \eta$ and $t_{2}=t_{0}-\rho^{\prime} \sigma \eta$. Then $0 \leq t_{1}<t_{2}<t_{0}$. We introduce further the shifted time $s=t-t_{1}$ and set $\tilde{f}(s)=\bar{f}\left(s+t_{1}\right), s \in\left(0, t_{0}-t_{1}\right)$, for functions $f$ defined on $\left(t_{1}, t_{0}\right)$. Since $u_{0} \geq 0$ in $B$ and $u$ is a positive weak supersolution of $(1.1)$ in $\left(0, t_{0}\right) \times B$, we have $(c f$. (3.3))

$$
\begin{array}{r}
\int_{B}\left(v \partial_{s}\left(g_{1-\alpha, n} * \tilde{u}\right)+\left(\left(h_{n} *[A D u]\right)^{\sim} \mid D v\right)\right) d x \geq 0, \\
\text { a.a. } s \in\left(0, t_{0}-t_{1}\right), n \in \mathbb{N},
\end{array}
$$

for any nonnegative function $v \in \stackrel{H}{H}_{2}^{1}(B)$. For $s \in\left(0, t_{0}-t_{1}\right)$ we choose the test function $v=\psi^{2} \tilde{u}^{\beta}$ with $\beta<-1$ and $\psi \in C_{0}^{1}\left(B_{1}\right)$ so that $0 \leq \psi \leq 1, \psi=1$ in $\rho^{\prime} B_{1}$, supp $\psi \subset \rho B_{1}$, and $|D \psi| \leq 2 /\left[\sigma\left(\rho-\rho^{\prime}\right)\right]$. By the fundamental identity (2.6) applied to $k=g_{1-\alpha, n}$ and the convex function $H(y)=-(1+\beta)^{-1} y^{1+\beta}$, $y>0$, there holds for a.a. $(s, x) \in\left(0, t_{0}-t_{1}\right) \times B$

$$
\begin{aligned}
-\tilde{u}^{\beta} \partial_{s}\left(g_{1-\alpha, n} * \tilde{u}\right) & \geq-\frac{1}{1+\beta} \partial_{s}\left(g_{1-\alpha, n} * \tilde{u}^{1+\beta}\right)+\left(\frac{\tilde{u}^{1+\beta}}{1+\beta}-\tilde{u}^{1+\beta}\right) g_{1-\alpha, n} \\
& =-\frac{1}{1+\beta} \partial_{s}\left(g_{1-\alpha, n} * \tilde{u}^{1+\beta}\right)-\frac{\beta}{1+\beta} \tilde{u}^{1+\beta} g_{1-\alpha, n} .
\end{aligned}
$$

We further have

$$
D v=2 \psi D \psi \tilde{u}^{\beta}+\beta \psi^{2} \tilde{u}^{\beta-1} D \tilde{u} .
$$

Using this and (3.6) it follows from (3.5) that for a.a. $s \in\left(0, t_{0}-t_{1}\right)$

$$
\begin{aligned}
& -\frac{1}{1+\beta} \int_{B_{1}} \psi^{2} \partial_{s}\left(g_{1-\alpha, n} * \tilde{u}^{1+\beta}\right) d x+|\beta| \int_{B_{1}}\left(\left(h_{n} *[A D u]\right)^{\sim} \mid \psi^{2} \tilde{u}^{\beta-1} D \tilde{u}\right) d x \\
& \leq 2 \int_{B_{1}}\left(\left(h_{n} *[A D u]\right)^{\sim} \mid \psi D \psi \tilde{u}^{\beta}\right) d x+\frac{\beta}{1+\beta} \int_{B_{1}} \psi^{2} \tilde{u}^{1+\beta} g_{1-\alpha, n} d x .
\end{aligned}
$$

Next, choose $\varphi \in C^{1}\left(\left[0, t_{0}-t_{1}\right]\right)$ such that $0 \leq \varphi \leq 1, \varphi=0$ in $\left[0,\left(t_{2}-t_{1}\right) / 2\right]$, $\varphi=1$ in $\left[t_{2}-t_{1}, t_{0}-t_{1}\right]$, and $0 \leq \dot{\varphi} \leq 4 /\left(t_{2}-t_{1}\right)$. Multiplying $(3.7)$ by $-(1+\beta)>0$ and by $\varphi(s)$, and convolving the resulting inequality with $g_{\alpha}$ yields

$$
\begin{aligned}
& \int_{B_{1}} g_{\alpha} *\left(\varphi \partial_{s}\left(g_{1-\alpha, n} *\left[\psi^{2} \tilde{u}^{1+\beta}\right]\right)\right) d x \\
& \quad+\beta(1+\beta) g_{\alpha} * \int_{B_{1}}\left(\left(h_{n} *[A D u]\right)^{\sim} \mid \psi^{2} \tilde{u}^{\beta-1} D \tilde{u}\right) \varphi d x \\
& \leq 2|1+\beta| g_{\alpha} * \int_{B_{1}}\left(\left(h_{n} *[A D u]\right)^{\sim} \mid \psi D \psi \tilde{u}^{\beta}\right) \varphi d x \\
& \quad+|\beta| g_{\alpha} * \int_{B_{1}} \psi^{2} \tilde{u}^{1+\beta} g_{1-\alpha, n} \varphi d x,
\end{aligned}
$$


for a.a. $s \in\left(0, t_{0}-t_{1}\right)$. By Lemma 2.4,

$$
\begin{aligned}
& \int_{B_{1}} g_{\alpha} *\left(\varphi \partial_{s}\left(g_{1-\alpha, n} *\left[\psi^{2} \tilde{u}^{1+\beta}\right]\right)\right) d x \geq \int_{B_{1}} \varphi g_{\alpha} *\left(\partial_{s}\left(g_{1-\alpha, n} *\left[\psi^{2} \tilde{u}^{1+\beta}\right]\right)\right) d x \\
& -\int_{0}^{s} g_{\alpha}(s-\sigma) \dot{\varphi}(\sigma)\left(g_{1-\alpha, n} * \int_{B_{1}} \psi^{2} \tilde{u}^{1+\beta} d x\right)(\sigma) d \sigma .
\end{aligned}
$$

Furthermore, by virtue of

$$
g_{1-\alpha, n} *\left[\psi^{2} \tilde{u}^{1+\beta}\right] \in{ }_{0} H_{1}^{1}\left(\left[0, t_{0}-t_{1}\right] ; L_{1}\left(B_{1}\right)\right)
$$

and $g_{1-\alpha, n}=g_{1-\alpha} * h_{n}$ as well as $g_{\alpha} * g_{1-\alpha}=1$ we have

$$
g_{\alpha} * \partial_{s}\left(g_{1-\alpha, n} *\left[\psi^{2} \tilde{u}^{1+\beta}\right]\right)=\partial_{s}\left(g_{\alpha} * g_{1-\alpha, n} *\left[\psi^{2} \tilde{u}^{1+\beta}\right]\right)=h_{n} *\left(\psi^{2} \tilde{u}^{1+\beta}\right) .
$$

Combining (3.8), (3.9), and (3.10), sending $n \rightarrow \infty$, and selecting an appropriate subsequence, if necessary, we thus obtain

$$
\begin{aligned}
& \int_{B_{1}} \varphi \psi^{2} \tilde{u}^{1+\beta} d x+\beta(1+\beta) g_{\alpha} * \int_{B_{1}}\left(\tilde{A} D \tilde{u} \mid \psi^{2} \tilde{u}^{\beta-1} D \tilde{u}\right) \varphi d x \\
& \leq 2|1+\beta| g_{\alpha} * \int_{B_{1}}\left(\tilde{A} D \tilde{u} \mid \psi D \psi \tilde{u}^{\beta}\right) \varphi d x+|\beta| g_{\alpha} * \int_{B_{1}} \psi^{2} \tilde{u}^{1+\beta} g_{1-\alpha} \varphi d x \\
& \quad+\int_{0}^{s} g_{\alpha}(s-\sigma) \dot{\varphi}(\sigma)\left(g_{1-\alpha} * \int_{B_{1}} \psi^{2} \tilde{u}^{1+\beta} d x\right)(\sigma) d \sigma, \text { a.a. } s \in\left(0, t_{0}-t_{1}\right) .
\end{aligned}
$$

Put $w=\tilde{u}^{\frac{\beta+1}{2}}$. Then $D w=\frac{\beta+1}{2} \tilde{u}^{\frac{\beta-1}{2}} D \tilde{u}$. By assumption $(\mathrm{H} 2)$, we have

$$
\begin{aligned}
& \beta(1+\beta) g_{\alpha} * \int_{B_{1}}\left(\tilde{A} D \tilde{u} \mid \psi^{2} \tilde{u}^{\beta-1} D \tilde{u}\right) \varphi d x \\
& \geq v \beta(1+\beta) g_{\alpha} * \int_{B_{1}} \varphi \psi^{2} \tilde{u}^{\beta-1}|D \tilde{u}|^{2} d x \\
& =\frac{4 v \beta}{1+\beta} g_{\alpha} * \int_{B_{1}} \varphi \psi^{2}|D w|^{2} d x .
\end{aligned}
$$

Using (H1) and Young's inequality we may estimate

$$
\begin{aligned}
2\left|\left(\tilde{A} D \tilde{u} \mid \psi D \psi \tilde{u}^{\beta}\right) \varphi\right| & \leq 2 \Lambda \psi|D \psi||D \tilde{u}| \tilde{u}^{\beta} \varphi=2 \Lambda \psi|D \psi||D \tilde{u}| \tilde{u}^{\frac{\beta-1}{2}} \tilde{u}^{\frac{\beta+1}{2}} \varphi \\
& \leq \frac{\nu|\beta|}{2} \psi^{2} \varphi|D \tilde{u}|^{2} \tilde{u}^{\beta-1}+\frac{2}{\nu|\beta|} \Lambda^{2}|D \psi|^{2} \varphi \tilde{u}^{\beta+1} \\
& =\frac{2 \nu|\beta|}{(1+\beta)^{2}} \psi^{2} \varphi|D w|^{2}+\frac{2}{\nu|\beta|} \Lambda^{2}|D \psi|^{2} \varphi w^{2}
\end{aligned}
$$


From (3.11), (3.12), and (3.13) we conclude that

$$
\int_{B_{1}} \varphi \psi^{2} w^{2} d x+\frac{2 v|\beta|}{|1+\beta|} g_{\alpha} * \int_{B_{1}} \varphi \psi^{2}|D w|^{2} d x \leq g_{\alpha} * F, \quad \text { a.a. } s \in\left(0, t_{0}-t_{1}\right),
$$

where

$$
\begin{aligned}
F(s)= & \frac{2 \Lambda^{2}|1+\beta|}{\nu|\beta|} \int_{B_{1}}|D \psi|^{2} \varphi w^{2} d x+|\beta| \varphi(s) g_{1-\alpha}(s) \int_{B_{1}} \psi^{2} w^{2} d x \\
& +\dot{\varphi}(s)\left(g_{1-\alpha} * \int_{B_{1}} \psi^{2} w^{2} d x\right)(s) \geq 0, \quad \text { a.a. } s \in\left(0, t_{0}-t_{1}\right) .
\end{aligned}
$$

We may drop the second term in (3.14), which is nonnegative. By Young's inequality for convolutions and the properties of $\varphi$ we then infer that for all $p \in$ $(1,1 /(1-\alpha))$

$$
\left(\int_{t_{2}-t_{1}}^{t_{0}-t_{1}}\left(\int_{B_{1}}[\psi(x) w(s, x)]^{2} d x\right)^{p} d s\right)^{1 / p} \leq\left|g_{\alpha}\right|_{L_{p}\left(\left[0, t_{0}-t_{1}\right]\right)} \int_{0}^{t_{0}-t_{1}} F(s) d s,
$$

where

$$
\begin{aligned}
\left|g_{\alpha}\right|_{L_{p}\left(\left[0, t_{0}-t_{1}\right]\right)} & =\frac{\left(t_{0}-t_{1}\right)^{\alpha-1+1 / p}}{\Gamma(\alpha)[(\alpha-1) p+1]^{1 / p}} \\
& \leq \frac{\eta^{\alpha-1+1 / p}}{\Gamma(\alpha)[(\alpha-1) p+1]^{1 / p}}=: C_{1}(\alpha, p, \eta) .
\end{aligned}
$$

We choose any of these $p$ and fix it.

Returning to (3.14), we may also drop the first term, convolve the resulting inequality with $g_{1-\alpha}$ and evaluate at $s=t_{0}-t_{1}$, thereby obtaining

$$
\int_{t_{2}-t_{1}}^{t_{0}-t_{1}} \int_{B_{1}} \psi^{2}|D w|^{2} d x d s \leq \frac{|1+\beta|}{2 v|\beta|} \int_{0}^{t_{0}-t_{1}} F(s) d s .
$$

Using

$$
\int_{t_{2}-t_{1}}^{t_{0}-t_{1}} \int_{B_{1}}|D(\psi w)|^{2} d x d s \leq 2 \int_{t_{2}-t_{1}}^{t_{0}-t_{1}} \int_{B_{1}}\left(\psi^{2}|D w|^{2}+|D \psi|^{2} w^{2}\right) d x d s
$$

we infer from (3.15)-(3.17) that

$$
\begin{aligned}
|\psi w|_{V_{p}\left(\left[t_{2}-t_{1}, t_{0}-t_{1}\right] \times B_{1}\right)}^{2} \leq & 2\left(C_{1}(\alpha, p, \eta)+\frac{|1+\beta|}{\nu|\beta|}\right) \int_{0}^{t_{0}-t_{1}} F(s) d s \\
& +4 \int_{0}^{t_{0}-t_{1}} \int_{B_{1}}|D \psi|^{2} w^{2} d x d s .
\end{aligned}
$$


We will next estimate the right-hand side of (3.18). By the assumptions on $\psi$ and $\varphi$, and since $|\beta|>1$, we have

$$
\int_{0}^{t_{0}-t_{1}} \int_{B_{1}}|D \psi|^{2} w^{2} d x d s \leq \frac{4}{\sigma^{2}\left(\rho-\rho^{\prime}\right)^{2}} \int_{0}^{t_{0}-t_{1}} \int_{\rho B_{1}} w^{2} d x d s
$$

and

$$
\begin{aligned}
F(s) \leq & \left(\frac{8 \Lambda^{2}|1+\beta|}{v \sigma^{2}\left(\rho-\rho^{\prime}\right)^{2}}+|\beta| g_{1-\alpha}\left(\left(t_{2}-t_{1}\right) / 2\right)\right) \int_{\rho B_{1}} w^{2} d x \\
& +\frac{4}{t_{2}-t_{1}}\left(g_{1-\alpha} * \int_{\rho B_{1}} w^{2} d x\right)(s), \quad \text { a.a. } s \in\left(0, t_{0}-t_{1}\right) .
\end{aligned}
$$

Recall that $\sigma \geq \delta>0$. So we have

$$
\begin{aligned}
\int_{0}^{t_{0}-t_{1}} F(s) d s \leq & \left(\frac{8 \Lambda^{2}|1+\beta|}{v \sigma^{2}\left(\rho-\rho^{\prime}\right)^{2}}+\frac{2^{\alpha}|\beta|}{\Gamma(1-\alpha)\left(\rho-\rho^{\prime}\right)^{\alpha}(\sigma \eta)^{\alpha}}\right) \int_{0}^{t_{0}-t_{1}} \int_{\rho B_{1}} w^{2} d x d s \\
& +\frac{4}{\left(\rho-\rho^{\prime}\right) \sigma \eta} \int_{0}^{t_{0}-t_{1}} g_{2-\alpha}\left(t_{0}-t_{1}-\tau\right) \int_{\rho B_{1}} w(\tau, x)^{2} d x d \tau \\
\leq & C(\nu, \Lambda, \delta, \eta, \alpha) \frac{1+|1+\beta|}{\left(\rho-\rho^{\prime}\right)^{2}} \int_{0}^{t_{0}-t_{1}} \int_{\rho B_{1}} w^{2} d x d s .
\end{aligned}
$$

Combining these estimates and (3.18) yields

$$
|\psi w|_{V_{p}\left(\left[t_{2}-t_{1}, t_{0}-t_{1}\right] \times B_{1}\right)} \leq C(\nu, \Lambda, \delta, \eta, \alpha, p) \frac{1+|1+\beta|}{\rho-\rho^{\prime}}|w|_{L_{2}\left(\left[0, t_{0}-t_{1}\right] \times \rho B_{1}\right)} .
$$

We apply next the interpolation inequality (2.9) to the function $\psi w$ and make use of $\psi=1$ in $\rho^{\prime} B_{1}$ to deduce that

$$
\begin{aligned}
& |w|_{L_{2 \kappa}\left(\left[t_{2}-t_{1}, t_{0}-t_{1}\right] \times \rho^{\prime} B_{1}\right)} \\
& \leq C(\nu, \Lambda, \delta, \eta, \alpha, p, N) \frac{1+|1+\beta|}{\rho-\rho^{\prime}}|w|_{L_{2}\left(\left[0, t_{0}-t_{1}\right] \times \rho B_{1}\right)},
\end{aligned}
$$

where the number $\kappa>1$ is given in (2.8). Since $w=\tilde{u}^{\frac{\beta+1}{2}}$ and by transforming back to the time $t$, we see that (3.19) is equivalent to

$$
\left(\int_{V_{\rho^{\prime}}} u^{-|1+\beta| \kappa} d \mu_{N+1}\right)^{\frac{1}{2 \kappa}} \leq \frac{\tilde{C}(1+|1+\beta|)}{\rho-\rho^{\prime}}\left(\int_{V_{\rho}} u^{-|1+\beta|} d \mu_{N+1}\right)^{\frac{1}{2}}
$$

with $\tilde{C}=\tilde{C}(v, \Lambda, \delta, \eta, \alpha, p, N)$. Hence, with $\gamma=|1+\beta|$,

$$
\left|u^{-1}\right|_{L_{\gamma \kappa}\left(V_{\rho^{\prime}}\right)} \leq\left(\frac{\tilde{C}^{2}(1+\gamma)^{2}}{\left(\rho-\rho^{\prime}\right)^{2}}\right)^{1 / \gamma}\left|u^{-1}\right|_{L_{\gamma}\left(V_{\rho}\right)}, \quad 0<\rho^{\prime}<\rho \leq 1, \gamma>0 .
$$


Employing the first Moser iteration, Lemma 2.1 (with $\bar{p}=1$ ), it follows that there exist constants $M_{0}=M_{0}(v, \Lambda, \delta, \eta, \alpha, p, N)$ and $\tau_{0}=\tau_{0}(\kappa)$ such that

$$
\underset{V_{\theta}}{\operatorname{ess} \sup } u^{-1} \leq\left(\frac{M_{0}}{(1-\theta)^{\tau_{0}}}\right)^{1 / \gamma}\left|u^{-1}\right|_{L_{\gamma}\left(V_{1}\right)} \quad \text { for all } \theta \in(0,1), \gamma \in(0,1]
$$

Thus if we take $\theta=\sigma^{\prime} / \sigma$ and notice that

$$
\frac{1}{1-\theta}=\frac{\sigma}{\sigma-\sigma^{\prime}} \leq \frac{1}{\sigma-\sigma^{\prime}}
$$

we obtain

$$
\underset{U_{\sigma^{\prime}}}{\operatorname{ess} \sup } u^{-1} \leq\left(\frac{M_{0}}{\left(\sigma-\sigma^{\prime}\right)^{\tau_{0}}}\right)^{1 / \gamma}\left|u^{-1}\right|_{L_{\gamma}\left(U_{\sigma}\right)}, \quad \gamma \in(0,1] .
$$

Hence the proof is complete.

We put

$$
\tilde{\kappa}:=\kappa_{1 /(1-\alpha)}=\frac{2+N \alpha}{2+N \alpha-2 \alpha} .
$$

Theorem 3.3. Let $\alpha \in(0,1), T>0$, and $\Omega \subset \mathbb{R}^{N}$ be a bounded domain. Suppose the assumptions (H1)-(H3) are satisfied. Let $\eta>0$ and $\delta \in(0,1)$ be fixed. Then for any $t_{0} \in[0, T)$ and $r>0$ with $t_{0}+\eta r^{2 / \alpha} \leq T$, any ball $B=B\left(x_{0}, r\right) \subset \Omega$, any $p_{0} \in(0, \tilde{\kappa})$, and any nonnegative weak supersolution $u$ of $(1.1)$ in $\left(0, t_{0}+\eta r^{2 / \alpha}\right) \times B$ with $u_{0} \geq 0$ in $B$, there holds

$$
\begin{aligned}
& |u|_{L_{p_{0}}\left(U_{\sigma^{\prime}}^{\prime}\right)} \leq\left(\frac{C \mu_{N+1}\left(U_{1}^{\prime}\right)^{-1}}{\left(\sigma-\sigma^{\prime}\right)^{\tau_{0}}}\right)^{1 / \gamma-1 / p_{0}}|u|_{L_{\gamma}\left(U_{\sigma}^{\prime}\right)}, \\
& \delta \leq \sigma^{\prime}<\sigma \leq 1,0<\gamma \leq p_{0} / \tilde{\kappa} .
\end{aligned}
$$

Here $U_{\sigma}^{\prime}=\left(t_{0}, t_{0}+\sigma \eta r^{2 / \alpha}\right) \times \sigma B, C=C\left(\nu, \Lambda, \delta, \eta, \alpha, N, p_{0}\right)$, and $\tau_{0}=$ $\tau_{0}(\alpha, N)$.

Proof. We proceed similarly as in the previous proof. Without restriction of generality we may assume that $p_{0}>1$ and $r=1$. By replacing $u$ with $u+\varepsilon$ and $u_{0}$ with $u_{0}+\varepsilon$ and eventually letting $\varepsilon \rightarrow 0+$ we may further assume that $u$ is bounded away from zero.

Fix $\sigma^{\prime}, \sigma$ such that $\delta \leq \sigma^{\prime}<\sigma \leq 1$ and put $B_{1}=\sigma B$. For $\rho \in(0,1]$ we set $V_{\rho}^{\prime}=U_{\rho \sigma}^{\prime}$. Given $0<\rho^{\prime}<\rho \leq 1$, let $t_{1}=t_{0}+\rho^{\prime} \sigma \eta$ and $t_{2}=t_{0}+\rho \sigma \eta$, so $0 \leq t_{0}<t_{1}<t_{2}$. We shift the time by means of $s=t-t_{0}$ and set $\tilde{f}(s)=f\left(s+t_{0}\right)$, $s \in\left(0, t_{2}-t_{0}\right)$, for functions $f$ defined on $\left(t_{0}, t_{2}\right)$. 
We then repeat the first steps of the preceding proof, the only difference being that now we take $\beta \in(-1,0)$. Note that, as a consequence of this, (3.6) simplifies to

$$
-\tilde{u}^{\beta} \partial_{s}\left(g_{1-\alpha, n} * \tilde{u}\right) \geq-\frac{1}{1+\beta} \partial_{s}\left(g_{1-\alpha, n} * \tilde{u}^{1+\beta}\right), \quad \text { a.a. }(s, x) \in\left(0, t_{2}-t_{0}\right) \times B,
$$

hence we obtain with $\psi \in C_{0}^{1}\left(B_{1}\right)$ as above

$$
\begin{aligned}
& \quad-\frac{1}{1+\beta} \int_{B_{1}} \psi^{2} \partial_{s}\left(g_{1-\alpha, n} * \tilde{u}^{1+\beta}\right) d x \\
& +|\beta| \int_{B_{1}}\left(\left(h_{n} *[A D u]\right)^{\sim} \mid \psi^{2} \tilde{u}^{\beta-1} D \tilde{u}\right) d x \\
& \leq 2 \int_{B_{1}}\left(\left(h_{n} *[A D u]\right)^{\sim} \mid \psi D \psi \tilde{u}^{\beta}\right) d x, \quad \text { a.a. } s \in\left(0, t_{2}-t_{0}\right) .
\end{aligned}
$$

Next, choose $\varphi \in C^{1}\left(\left[0, t_{2}-t_{0}\right]\right)$ such that $0 \leq \varphi \leq 1, \varphi=1$ in $\left[0, t_{1}-t_{0}\right], \varphi=0$ in $\left[t_{1}-t_{0}+\left(t_{2}-t_{1}\right) / 2, t_{2}-t_{0}\right]$, and $0 \leq-\dot{\varphi} \leq 4 /\left(t_{2}-t_{1}\right)$. Multiplying (3.20) by $1+\beta>0$ and by $\varphi(s)$, and applying Lemma 2.5 to the first term gives

$$
\begin{aligned}
& -\int_{B_{1}} \partial_{s}\left(g_{1-\alpha, n} *\left[\varphi \psi^{2} \tilde{u}^{1+\beta}\right]\right) d x+|\beta|(1+\beta) \int_{B_{1}}\left(\tilde{A} D \tilde{u} \mid \psi^{2} \tilde{u}^{\beta-1} D \tilde{u}\right) \varphi d x \\
\leq & \int_{0}^{s} \dot{g}_{1-\alpha, n}(s-\sigma)(\varphi(s)-\varphi(\sigma))\left(\int_{B_{1}} \psi^{2} \tilde{u}^{1+\beta} d x\right)(\sigma) d \sigma \\
& +2(1+\beta) \int_{B_{1}}\left(\tilde{A} D \tilde{u} \mid \psi D \psi \tilde{u}^{\beta}\right) \varphi d x+\mathcal{R}_{n}(s), \quad \text { a.a. } s \in\left(0, t_{2}-t_{0}\right),
\end{aligned}
$$

where

$$
\begin{aligned}
\mathcal{R}_{n}(s)= & -|\beta|(1+\beta) \int_{B_{1}}\left(\left(h_{n} *[A D u]\right)^{\sim}-\tilde{A} D \tilde{u} \mid \psi^{2} \tilde{u}^{\beta-1} D \tilde{u}\right) \varphi d x \\
& +2(1+\beta) \int_{B_{1}}\left(\left(h_{n} *[A D u]\right)^{\sim}-\tilde{A} D \tilde{u} \mid \psi D \psi \tilde{u}^{\beta}\right) \varphi d x, \text { a.a. } s \in\left(0, t_{2}-t_{0}\right) .
\end{aligned}
$$

We set again $w=\tilde{u}^{\frac{\beta+1}{2}}$ and estimate exactly as in the preceding proof, using (H1), (H3) and (3.13), to the result

$$
\begin{aligned}
& -\int_{B_{1}} \partial_{s}\left(g_{1-\alpha, n} *\left[\varphi \psi^{2} w^{2}\right]\right) d x+\frac{2 \nu|\beta|}{1+\beta} \int_{B_{1}} \varphi \psi^{2}|D w|^{2} d x \\
\leq & \int_{0}^{s} \dot{g}_{1-\alpha, n}(s-\sigma)(\varphi(s)-\varphi(\sigma))\left(\int_{B_{1}} \psi^{2} w^{2} d x\right)(\sigma) d \sigma \\
& +\frac{2 \Lambda^{2}(1+\beta)}{\nu|\beta|} \int_{B_{1}}|D \psi|^{2} \varphi w^{2} d x+\mathcal{R}_{n}(s), \quad \text { a.a. } s \in\left(0, t_{2}-t_{0}\right) .
\end{aligned}
$$


Recall that $g_{1-\alpha, n}=g_{1-\alpha} * h_{n}$. Putting

$$
W(s)=\int_{B_{1}} \varphi(s) \psi(x)^{2} w(s, x)^{2} d x
$$

and denoting the right-hand side of (3.22) by $F_{n}(s)$, it follows from (3.22) that

$$
G_{n}(s):=\partial_{s}^{\alpha}\left(h_{n} * W\right)(s)+F_{n}(s) \geq 0, \quad \text { a.a. } s \in\left(0, t_{2}-t_{0}\right) .
$$

By (3.10) and positivity of $h_{n}$, we have

$$
0 \leq h_{n} * W=g_{\alpha} * \partial_{s}^{\alpha}\left(h_{n} * W\right) \leq g_{\alpha} * G_{n}+g_{\alpha} *\left[-F_{n}(s)\right]_{+}
$$

a.e. in $\left(0, t_{2}-t_{0}\right)$, where $[y]_{+}$stands for the positive part of $y \in \mathbb{R}$. For any $p \in(1,1 /(1-\alpha))$ and any $t_{*} \in\left[t_{2}-t_{0}-\left(t_{2}-t_{1}\right) / 4, t_{2}-t_{0}\right]$ we thus obtain by Young's inequality

$$
\left|h_{n} * W\right|_{L_{p}\left(\left[0, t_{*}\right]\right)} \leq\left|g_{\alpha}\right|_{L_{p}\left(\left[0, t_{*}\right]\right)}\left(\left|G_{n}\right|_{L_{1}\left(\left[0, t_{*}\right]\right)}+\left|\left[-F_{n}\right]_{+}\right|_{L_{1}\left(\left[0, t_{*}\right]\right)}\right) .
$$

Since $t_{*} \leq t_{2}-t_{0} \leq \eta$, we have $\left|g_{\alpha}\right|_{L_{p}\left(\left[0, t_{*}\right]\right)} \leq C_{1}(\alpha, p, \eta)$ with the same constant as in (3.16). By positivity of $G_{n}$,

$$
\left|G_{n}\right|_{L_{1}\left(\left[0, t_{*}\right]\right)}=\left(g_{1-\alpha, n} * W\right)\left(t_{*}\right)+\int_{0}^{t_{*}} F_{n}(s) d s .
$$

Observe that $\mathcal{R}_{n} \rightarrow 0$ in $L_{1}\left(\left[0, t_{2}-t_{0}\right]\right)$ as $n \rightarrow \infty$. Hence $\left|\left[-F_{n}\right]_{+}\right|_{L_{1}\left(\left[0, t_{*}\right]\right)} \rightarrow 0$ as $n \rightarrow \infty$. Further,

$$
\begin{gathered}
\int_{0}^{t_{*}} \int_{0}^{s} \dot{g}_{1-\alpha, n}(s-\sigma)(\varphi(s)-\varphi(\sigma))\left(\int_{B_{1}} \psi^{2} w^{2} d x\right)(\sigma) d \sigma d s \\
=\int_{0}^{t_{*}} g_{1-\alpha, n}\left(t_{*}-\sigma\right)\left(\varphi\left(t_{*}\right)-\varphi(\sigma)\right)\left(\int_{B_{1}} \psi^{2} w^{2} d x\right)(\sigma) d \sigma \\
\quad-\int_{0}^{t_{*}} \dot{\varphi}(s) \int_{0}^{s} g_{1-\alpha, n}(s-\sigma)\left(\int_{B_{1}} \psi^{2} w^{2} d x\right)(\sigma) d \sigma d s \\
\leq-\int_{0}^{t_{*}} \dot{\varphi}(s) \int_{0}^{s} g_{1-\alpha, n}(s-\sigma)\left(\int_{B_{1}} \psi^{2} w^{2} d x\right)(\sigma) d \sigma d s,
\end{gathered}
$$

since $\varphi$ is nonincreasing. We also know that $g_{1-\alpha, n} * W \rightarrow g_{1-\alpha} * W$ in $L_{1}\left(\left[0, t_{2}-\right.\right.$ $\left.\left.t_{0}\right]\right)$. Hence we can fix some $t_{*} \in\left[t_{2}-t_{0}-\left(t_{2}-t_{1}\right) / 4, t_{2}-t_{0}\right]$ such that for some subsequence $\left(g_{1-\alpha, n_{k}} * W\right)\left(t_{*}\right) \rightarrow\left(g_{1-\alpha} * W\right)\left(t_{*}\right)$ as $k \rightarrow \infty$. Sending $k \rightarrow \infty$ it follows then from (3.23), the preceding estimates, and from $\varphi=1$ in $\left[0, t_{1}-t_{0}\right]$ that

$$
\begin{aligned}
& \left(\int_{0}^{t_{1}-t_{0}}\left(\int_{B_{1}}[\psi(x) w(s, x)]^{2} d x\right)^{p} d s\right)^{1 / p} \\
& \leq C_{1}(\alpha, p, \eta)\left(\left(g_{1-\alpha} * W\right)\left(t_{*}\right)+|F|_{L_{1}\left(\left[0, t_{2}-t_{0}\right]\right)}\right),
\end{aligned}
$$


with

$$
F(s)=\frac{2 \Lambda^{2}(1+\beta)}{\nu|\beta|} \int_{B_{1}}|D \psi|^{2} \varphi w^{2} d x-\dot{\varphi}(s)\left(g_{1-\alpha} * \int_{B_{1}} \psi^{2} w^{2} d x\right)(s) .
$$

On the other hand, we can integrate (3.22) over $\left(0, t_{*}\right)$ and take the limit as $k \rightarrow \infty$ for the same subsequence as before, thereby getting

$$
\int_{0}^{t_{1}-t_{0}} \int_{B_{1}} \psi^{2}|D w|^{2} d x d s \leq \frac{1+\beta}{2 \nu|\beta|}\left(\left(g_{1-\alpha} * W\right)\left(t_{*}\right)+|F|_{L_{1}\left(\left[0, t_{2}-t_{0}\right]\right)}\right) .
$$

Arguing as above ( $c f$. the lines before (3.18)), we conclude from (3.24) and (3.25) that

$$
\begin{aligned}
& |\psi w|_{V_{p}\left(\left[0, t_{1}-t_{0}\right] \times B_{1}\right)}^{2} \leq 4 \int_{0}^{t_{2}-t_{0}} \int_{B_{1}}|D \psi|^{2} w^{2} d x d s \\
& +2\left(C_{1}(\alpha, p, \eta)+\frac{1+\beta}{\nu|\beta|}\right)\left(\left(g_{1-\alpha} * W\right)\left(t_{*}\right)+|F|_{L_{1}\left(\left[0, t_{2}-t_{0}\right]\right)}\right) .
\end{aligned}
$$

Since $\varphi=0$ in $\left[t_{1}-t_{0}+\left(t_{2}-t_{1}\right) / 2, t_{2}-t_{0}\right]$ and $t_{*} \in\left[t_{2}-t_{0}-\left(t_{2}-t_{1}\right) / 4, t_{2}-t_{0}\right]$, we have

$$
\begin{aligned}
\left(g_{1-\alpha} * W\right)\left(t_{*}\right) & \leq g_{1-\alpha}\left(\left(t_{2}-t_{1}\right) / 4\right) \int_{0}^{t_{2}-t_{0}} \int_{B_{1}} \psi^{2} w^{2} d x d s \\
& =\frac{4^{\alpha}}{\Gamma(1-\alpha)\left(\rho-\rho^{\prime}\right)^{\alpha}(\sigma \eta)^{\alpha}} \int_{0}^{t_{2}-t_{0}} \int_{\rho B_{1}} w^{2} d x d s .
\end{aligned}
$$

Further,

$$
\int_{0}^{t_{2}-t_{0}} \int_{B_{1}}|D \psi|^{2} w^{2} d x d s \leq \frac{4}{\sigma^{2}\left(\rho-\rho^{\prime}\right)^{2}} \int_{0}^{t_{2}-t_{0}} \int_{\rho B_{1}} w^{2} d x d s .
$$

The term $|F|_{L_{1}\left(\left[0, t_{2}-t_{0}\right]\right)}$ is estimated similarly as in the proof of Theorem 3.2 (cf. the lines that follow (3.18)). We obtain

$$
|F|_{L_{1}\left(\left[0, t_{2}-t_{0}\right]\right)} \leq C(\nu, \Lambda, \delta, \eta, \alpha) \frac{1+(1+\beta)}{|\beta|\left(\rho-\rho^{\prime}\right)^{2}} \int_{0}^{t_{2}-t_{0}} \int_{\rho B_{1}} w^{2} d x d s .
$$

Notice the additional factor $|\beta|$ in the denominator. Combining these estimates we deduce from (3.26) that

$$
|\psi w|_{V_{p}\left(\left[0, t_{1}-t_{0}\right] \times B_{1}\right)} \leq C(\nu, \Lambda, \delta, \eta, \alpha, p) \frac{1+(1+\beta)}{|\beta|\left(\rho-\rho^{\prime}\right)}|w|_{L_{2}\left(\left[0, t_{2}-t_{0}\right] \times \rho B_{1}\right)} .
$$


By the interpolation inequality (2.9) and since $\psi=1$ in $\rho^{\prime} B_{1}$, this implies for all $\beta \in(-1,0)$

$$
\begin{aligned}
& |w|_{L_{2 \kappa}\left(\left[0, t_{1}-t_{0}\right] \times \rho^{\prime} B_{1}\right)} \\
& \leq C(v, \Lambda, \delta, \eta, \alpha, p, N) \frac{1+|1+\beta|}{|\beta|\left(\rho-\rho^{\prime}\right)}|w|_{L_{2}\left(\left[0, t_{2}-t_{0}\right] \times \rho B_{1}\right)},
\end{aligned}
$$

where

$$
\kappa=\kappa_{p}=\frac{2 p+N(p-1)}{2+N(p-1)} \in(1, \tilde{\kappa}) .
$$

We now fix $1<p<1 /(1-\alpha)$ such that $\kappa_{p}=\left(p_{0}+\tilde{\kappa}\right) / 2$. This is possible because $\kappa_{p} \nearrow \tilde{\kappa}$ as $p \nearrow 1 /(1-\alpha)$.

Next, we set $\gamma=1+\beta \in(0,1)$ and transform back to $u$ to get

$$
\begin{aligned}
& |u|_{L_{\gamma \kappa}\left(V_{\rho^{\prime}}^{\prime}, d \mu\right)} \leq\left(\frac{\tilde{C}}{\left(\rho-\rho^{\prime}\right)^{2}}\right)^{1 / \gamma}|u|_{L_{\gamma}\left(V_{\rho}^{\prime}, d \mu\right)}, \\
& 0<\rho^{\prime}<\rho \leq 1,0<\gamma \leq p_{0} / \kappa .
\end{aligned}
$$

Here, $\mu=\left(\eta \omega_{N}\right)^{-1} \mu_{N+1}, \omega_{N}$ the volume of the unit ball in $\mathbb{R}^{N}$, and $\tilde{C}=$ $\tilde{C}\left(\nu, \Lambda, \delta, \eta, \alpha, N, p_{0}\right)$ is independent of $\gamma \in\left(0, p_{0} / \kappa\right]$, since $|\beta|$ is bounded away from zero. Note that $\mu\left(V_{1}^{\prime}\right) \leq 1$.

Finally, we employ the second Moser iteration scheme, Lemma 2.2, to conclude from (3.28) that there are constants $M_{0}=M_{0}\left(\nu, \Lambda, \delta, \eta, \alpha, N, p_{0}\right)$ and $\tau_{0}=$ $\tau_{0}(\kappa)$ such that

$$
\begin{aligned}
& |u|_{L_{p_{0}}\left(V_{\theta}^{\prime}, d \mu\right)} \leq\left(\frac{M_{0}}{(1-\theta)^{\tau_{0}}}\right)^{1 / \gamma-1 / p_{0}}|u|_{L_{\gamma}\left(V_{1}^{\prime}, d \mu\right)}, \\
& 0<\theta<1,0<\gamma \leq p_{0} / \kappa
\end{aligned}
$$

If we take $\theta=\sigma^{\prime} / \sigma$ and translate (3.29) back to the measure $\mu_{N+1}$, we obtain

$$
|u|_{L_{p_{0}}\left(U_{\sigma^{\prime}}^{\prime}\right)} \leq\left(\frac{M_{0}\left(\eta \omega_{N}\right)^{-1}}{\left(\sigma-\sigma^{\prime}\right)^{\tau_{0}}}\right)^{1 / \gamma-1 / p_{0}}|u|_{L_{\gamma}\left(U_{\sigma}^{\prime}\right)}, \quad 0<\gamma \leq p_{0} / \kappa .
$$

Since $\kappa<\tilde{\kappa},(3.30)$ holds in particular for all $\gamma \in\left(0, p_{0} / \tilde{\kappa}\right]$. This finishes the proof.

\subsection{Logarithmic estimates}

Theorem 3.4. Let $\alpha \in(0,1), T>0$, and $\Omega \subset \mathbb{R}^{N}$ be a bounded domain. Suppose the assumptions $(\mathrm{H} 1)-(\mathrm{H} 3)$ are satisfied. Let $\tau>0$ and $\delta, \eta \in(0,1)$ be fixed. Then for any $t_{0} \geq 0$ and $r>0$ with $t_{0}+\tau r^{2 / \alpha} \leq T$, any ball $B=B\left(x_{0}, r\right) \subset \Omega$, and 
any weak supersolution $u \geq \varepsilon>0$ of $(1.1)$ in $\left(0, t_{0}+\tau r^{2 / \alpha}\right) \times B$ with $u_{0} \geq 0$ in $B$, there is a constant $c=c(u)$ such that

$$
\mu_{N+1}\left(\left\{(t, x) \in K_{-}: \log u(t, x)>c+\lambda\right\}\right) \leq C r^{2 / \alpha} \mu_{N}(B) \lambda^{-1}, \quad \lambda>0,
$$

and

$$
\mu_{N+1}\left(\left\{(t, x) \in K_{+}: \log u(t, x)<c-\lambda\right\}\right) \leq C r^{2 / \alpha} \mu_{N}(B) \lambda^{-1}, \quad \lambda>0,
$$

where $K_{-}=\left(t_{0}, t_{0}+\eta \tau r^{2 / \alpha}\right) \times \delta B$ and $K_{+}=\left(t_{0}+\eta \tau r^{2 / \alpha}, t_{0}+\tau r^{2 / \alpha}\right) \times \delta B$. Here the constant $C$ depends only on $\delta, \eta, \tau, N, \alpha, v$, and $\Lambda$.

Proof. Since $u_{0} \geq 0$ in $B$ and $u$ is a positive weak supersolution we may assume without loss of generality that $u_{0}=0$ and $t_{0}=0$. In fact, in the case $t_{0}>0$ we shift the time as $t \rightarrow t-t_{0}$, thereby obtaining an inequality of the same type on the time-interval $J:=\left[0, \tau r^{2 / \alpha}\right]$. Observe that the property $g_{1-\alpha} * u \in C\left(\left[0, t_{0}+\right.\right.$ $\left.\left.\tau r^{2 / \alpha}\right] ; L_{2}(B)\right)$ implies $g_{1-\alpha} * \tilde{u} \in C\left(J ; L_{2}(B)\right)$ for the shifted function $\tilde{u}(s, x)=$ $u\left(s+t_{0}, x\right)$. So we have

$$
\int_{B}\left(v \partial_{t}\left(g_{1-\alpha, n} * u\right)+\left(h_{n} *[A D u] \mid D v\right)\right) d x \geq 0, \quad \text { a.a. } t \in J, n \in \mathbb{N},
$$

for any nonnegative test function $v \in \stackrel{\circ}{H}_{2}^{1}(B)$.

For $t \in J$ we choose the test function $v=\psi^{2} u^{-1}$ with $\psi \in C_{0}^{1}(B)$ such that $\operatorname{supp} \psi \subset B, \psi=1$ in $\delta B, 0 \leq \psi \leq 1,|D \psi| \leq 2 /[(1-\delta) r]$ and the domains $\left\{x \in B: \psi(x)^{2} \geq b\right\}$ are convex for all $b \leq 1$. We have

$$
D v=2 \psi D \psi u^{-1}-\psi^{2} u^{-2} D u,
$$

so that by substitution into (3.33) we obtain for a.a. $t \in J$

$$
\begin{aligned}
& -\int_{B} \psi^{2} u^{-1} \partial_{t}\left(g_{1-\alpha, n} * u\right) d x+\int_{B}\left(A D u \mid u^{-2} D u\right) \psi^{2} d x \\
\leq & 2 \int_{B}\left(A D u \mid u^{-1} \psi D \psi\right) d x+\mathcal{R}_{n}(t),
\end{aligned}
$$

where

$$
\mathcal{R}_{n}(t)=\int_{B}\left(h_{n} *[A D u]-A D u \mid D v\right) d x .
$$

By (H1) and Young's inequality,

$$
\left|2\left(A D u \mid u^{-1} \psi D \psi\right)\right| \leq 2 \Lambda \psi|D \psi||D u| u^{-1} \leq \frac{v}{2} \psi^{2}|D u|^{2} u^{-2}+\frac{2}{v} \Lambda^{2}|D \psi|^{2} .
$$

Using this, (H2) and $|D \psi| \leq 2 /[(1-\delta) r]$, we infer from (3.34) that for a.a. $t \in J$

$$
\begin{aligned}
& -\int_{B} \psi^{2} u^{-1} \partial_{t}\left(g_{1-\alpha, n} * u\right) d x \\
& +\frac{v}{2} \int_{B}|D u|^{2} u^{-2} \psi^{2} d x \leq \frac{8 \Lambda^{2} \mu_{N}(B)}{v(1-\delta)^{2} r^{2}}+\mathcal{R}_{n}(t) .
\end{aligned}
$$


Setting $w=\log u$ we have $D w=u^{-1} D u$. The weighted Poincaré inequality of Proposition 2.6 with weight $\psi^{2}$ yields

$$
\int_{B}(w-W)^{2} \psi^{2} d x \leq \frac{8 r^{2} \mu_{N}(B)}{\int_{B} \psi^{2} d x} \int_{B}|D w|^{2} \psi^{2} d x, \quad \text { a.a. } t \in J
$$

where

$$
W(t)=\frac{\int_{B} w(t, x) \psi(x)^{2} d x}{\int_{B} \psi(x)^{2} d x}, \quad \text { a.a. } t \in J .
$$

From (3.35) and (3.36) we deduce that

$$
-\int_{B} \psi^{2} u^{-1} \partial_{t}\left(g_{1-\alpha, n} * u\right) d x+\frac{v \int_{B} \psi^{2} d x}{16 r^{2} \mu_{N}(B)} \int_{B}(w-W)^{2} \psi^{2} d x \leq \frac{8 \Lambda^{2} \mu_{N}(B)}{v(1-\delta)^{2} r^{2}}+\mathcal{R}_{n}(t),
$$

which in turn implies

$$
\begin{aligned}
& \frac{-\int_{B} \psi^{2} u^{-1} \partial_{t}\left(g_{1-\alpha, n} * u\right) d x}{\int_{B} \psi^{2} d x} \\
& +\frac{v}{16 r^{2} \mu_{N}(B)} \int_{\delta B}(w-W)^{2} d x \leq \frac{C_{1}}{r^{2}}+S_{n}(t),
\end{aligned}
$$

for a.a. $t \in J$, with some constant $C_{1}=C_{1}(\delta, N, v, \Lambda)$ and $S_{n}(t)=\mathcal{R}_{n}(t) / \int_{B} \psi^{2} d x$.

The fundamental identity (2.6) with $H(y)=-\log y$ reads (with the spatial variable $x$ being suppressed)

$$
\begin{aligned}
& -u^{-1} \partial_{t}\left(g_{1-\alpha, n} * u\right)=-\partial_{t}\left(g_{1-\alpha, n} * \log u\right)+(\log u-1) g_{1-\alpha, n}(t) \\
& +\int_{0}^{t}\left(-\log u(t-s)+\log u(t)+\frac{u(t-s)-u(t)}{u(t)}\right)\left[-\dot{g}_{1-\alpha, n}(s)\right] d s .
\end{aligned}
$$

In terms of $w=\log u$ this means that

$$
\begin{aligned}
-u^{-1} \partial_{t}\left(g_{1-\alpha, n} * u\right)= & -\partial_{t}\left(g_{1-\alpha, n} * w\right)+(w-1) g_{1-\alpha, n}(t) \\
& +\int_{0}^{t} \Psi(w(t-s)-w(t))\left[-\dot{g}_{1-\alpha, n}(s)\right] d s,
\end{aligned}
$$

where $\Psi(y)=e^{y}-1-y$. Since $\Psi$ is convex, it follows from Jensen's inequality that

$$
\frac{\int_{B} \psi^{2} \Psi(w(t-s, x)-w(t, x)) d x}{\int_{B} \psi^{2} d x} \geq \Psi\left(\frac{\int_{B} \psi^{2}(w(t-s, x)-w(t, x)) d x}{\int_{B} \psi^{2} d x}\right) .
$$


Using this and (3.38) we obtain

$$
\begin{aligned}
\frac{-\int_{B} \psi^{2} u^{-1} \partial_{t}\left(g_{1-\alpha, n} * u\right) d x}{\int_{B} \psi^{2} d x} \geq & -\partial_{t}\left(g_{1-\alpha, n} * W\right)+(W-1) g_{1-\alpha, n}(t) \\
& +\int_{0}^{t} \Psi(W(t-s)-W(t))\left[-\dot{g}_{1-\alpha, n}(s)\right] d s \\
= & -e^{-W} \partial_{t}\left(g_{1-\alpha, n} * e^{W}\right),
\end{aligned}
$$

where the last equals sign holds again by (3.38) with $u$ replaced by $e^{W}$. From (3.37) and (3.39) we conclude that

$$
\frac{v}{16 r^{2} \mu_{N}(B)} \int_{\delta B}(w-W)^{2} d x \leq e^{-W} \partial_{t}\left(g_{1-\alpha, n} * e^{W}\right)+\frac{C_{1}}{r^{2}}+S_{n}(t), \quad \text { a.a. } t \in J
$$

We choose

$$
c(u)=\log \left(\frac{\left(g_{1-\alpha} * e^{W}\right)\left(\eta \tau r^{2 / \alpha}\right)}{g_{2-\alpha}\left(\eta \tau r^{2 / \alpha}\right)}\right) .
$$

This definition makes sense, since $g_{1-\alpha} * e^{W} \in C(J)$. The latter is a consequence of $g_{1-\alpha} * u \in C\left(J ; L_{2}(B)\right)$ and

$$
e^{W(t)} \leq \frac{\int_{B} u(t, x) \psi(x)^{2} d x}{\int_{B} \psi(x)^{2} d x}, \quad \text { a.a. } t \in J
$$

where we apply again Jensen's inequality.

To prove (3.31) and (3.32), one of the key ideas is to use the inequalities

$$
\begin{aligned}
& \mu_{N+1}\left(\left\{(t, x) \in K_{-}: w(t, x)>c(u)+\lambda\right\}\right) \\
& \leq \quad \mu_{N+1}\left(\left\{(t, x) \in K_{-}: w(t, x)>c(u)+\lambda \text { and } W(t) \leq c(u)+\lambda / 2\right\}\right) \\
& \quad+\mu_{N+1}\left(\left\{(t, x) \in K_{-}: W(t)>c(u)+\lambda / 2\right\}\right)=: I_{1}+I_{2}, \quad \lambda>0, \\
& \mu_{N+1}\left(\left\{(t, x) \in K_{+}: w(t, x)<c(u)-\lambda\right\}\right) \\
& \leq \mu_{N+1}\left(\left\{(t, x) \in K_{+}: w(t, x)<c(u)-\lambda \text { and } W(t) \geq c(u)-\lambda / 2\right\}\right) \\
& \quad+\mu_{N+1}\left(\left\{(t, x) \in K_{+}: W(t)<c(u)-\lambda / 2\right\}\right)=: I_{3}+I_{4}, \quad \lambda>0,
\end{aligned}
$$

and to estimate each of the four terms $I_{j}$ separately.

We begin with the estimates for $W$. To estimate $I_{2}$ and $I_{4}$ we adopt some of the ideas developed in [32]. We set $J_{-}:=\left(0, \eta \tau r^{2 / \alpha}\right), J_{+}:=\left(\eta \tau r^{2 / \alpha}, \tau r^{2 / \alpha}\right)$, and introduce for $\lambda>0$ the sets $J_{-}(\lambda):=\left\{t \in J_{-}: W(t)>c(u)+\lambda\right\}$ and $J_{+}(\lambda):=\left\{t \in J_{+}: W(t)<c(u)-\lambda\right\}$.

Interestingly, positivity and integrability of the function $e^{W}$ are sufficient to derive the desired estimate for $I_{2}, c f$. also [32, Theorem 2.3]. In fact, with $\rho=$ 
$\tau r^{2 / \alpha}$ we have

$$
\begin{aligned}
e^{\lambda} \mu_{1}\left(J_{-}(\lambda)\right) & =e^{\lambda} \mu_{1}\left(\left\{t \in J_{-}: e^{W(t)}>e^{c(u)} e^{\lambda}\right\}\right)=\int_{J_{-}(\lambda)} e^{\lambda} d t \\
& \leq \int_{J_{-}(\lambda)} e^{W(t)-c(u)} d t \leq \int_{J_{-}} e^{W(t)-c(u)} d t \\
& =\frac{g_{2-\alpha}(\eta \rho)}{\left(g_{1-\alpha} * e^{W}\right)(\eta \rho)} \int_{0}^{\eta \rho} e^{W(t)} d t \\
& \leq \frac{g_{2-\alpha}(\eta \rho)}{\left(g_{1-\alpha} * e^{W}\right)(\eta \rho)} \cdot \frac{1}{g_{1-\alpha}(\eta \rho)} \int_{0}^{\eta \rho} g_{1-\alpha}(\eta \rho-t) e^{W(t)} d t \\
& =\frac{\Gamma(1-\alpha)}{\Gamma(2-\alpha)} \eta \rho=\frac{\eta \tau r^{2 / \alpha}}{1-\alpha},
\end{aligned}
$$

and therefore

$$
I_{2}=\mu_{1}\left(J_{-}(\lambda / 2)\right) \mu_{N}(\delta B) \leq \frac{2 \eta \tau \delta^{N}}{(1-\alpha) \lambda} r^{2 / \alpha} \mu_{N}(B), \quad \lambda>0 .
$$

We come now to $I_{4}$. For $m>0$ define the function $H_{m}$ on $\mathbb{R}$ by $H_{m}(y)=y$, $y \leq m$, and $H_{m}(y)=m+(y-m) /(y-m+1), y \geq m$. Then $H_{m}$ is increasing, concave, and bounded above by $m+1$. Further, we have $H_{m} \in C^{1}(\mathbb{R})$, and so by concavity

$$
0 \leq y H_{m}^{\prime}(y) \leq H_{m}(y) \leq m+1, \quad y \geq 0 .
$$

Multiplying (3.40) by $e^{W} H_{m}^{\prime}\left(e^{W}\right)$ and employing (3.45) as well as the fundamental identity (2.6), we infer that

$$
\partial_{t}\left(g_{1-\alpha, n} * H_{m}\left(e^{W}\right)\right)+\frac{C_{1}}{r^{2}} H_{m}\left(e^{W}\right) \geq-S_{n} e^{W} H_{m}^{\prime}\left(e^{W}\right), \quad \text { a.a. } t \in J .
$$

For $t \in J_{+}$we shift the time by setting $s=t-\eta \tau r^{2 / \alpha}=t-\eta \rho$ and put $\tilde{f}(s)=$ $f(s+\eta \rho), s \in(0,(1-\eta) \rho)$, for functions $f$ defined on $J_{+}$. By the time-shifting identity (3.2), (3.46) implies that for a.a. $s \in(0,(1-\eta) \rho)$

$$
\partial_{s}\left(g_{1-\alpha, n} * H_{m}\left(e^{\tilde{W}}\right)\right)+\frac{C_{1}}{r^{2}} H_{m}\left(e^{\tilde{W}}\right) \geq \Upsilon_{n, m}(s)-\tilde{S}_{n} e^{\tilde{W}} H_{m}^{\prime}\left(e^{\tilde{W}}\right),
$$

with the history term

$$
\Upsilon_{n, m}(s)=\int_{0}^{\eta \rho}\left[-\dot{g}_{1-\alpha, n}(s+\eta \rho-\sigma)\right] H_{m}\left(e^{W(\sigma)}\right) d \sigma .
$$

For $\theta \geq 0$ define the kernel $r_{\alpha, \theta} \in L_{1, \text { loc }}\left(\mathbb{R}_{+}\right)$by means of

$$
r_{\alpha, \theta}(t)+\theta\left(r_{\alpha, \theta} * g_{\alpha}\right)(t)=g_{\alpha}(t), \quad t>0 .
$$


Observe that $r_{\alpha, 0}=g_{\alpha}$. Since $g_{\alpha}$ is completely monotone, $r_{\alpha, \theta}$ enjoys the same property ( $c f$. [13, Chapter 5]), in particular $r_{\alpha, \theta}(s)>0$ for all $s>0$. Moreover, we have (see e.g. [32])

$$
r_{\alpha, \theta}(s)=\Gamma(\alpha) g_{\alpha}(s) E_{\alpha, \alpha}\left(-\theta s^{\alpha}\right), \quad s>0,
$$

where $E_{\alpha, \beta}$ denotes the generalized Mittag-Leffler-function defined by

$$
E_{\alpha, \beta}(z)=\sum_{n=0}^{\infty} \frac{z^{n}}{\Gamma(n \alpha+\beta)}, \quad z \in \mathbb{C} .
$$

We put $\theta=C_{1} / r^{2}$ and convolve (3.47) with $r_{\alpha, \theta}$. We have a.e. in $(0,(1-\eta) \rho)$

$$
\begin{aligned}
r_{\alpha, \theta} * \partial_{s}\left(g_{1-\alpha, n} * H_{m}\left(e^{\tilde{W}}\right)\right) & =\partial_{s}\left(r_{\alpha, \theta} * g_{1-\alpha, n} * H_{m}\left(e^{\tilde{W}}\right)\right) \\
& =\partial_{s}\left(\left[g_{\alpha}-\theta\left(r_{\alpha, \theta} * g_{\alpha}\right)\right] * g_{1-\alpha, n} * H_{m}\left(e^{\tilde{W}}\right)\right) \\
& =h_{n} * H_{m}\left(e^{\tilde{W}}\right)-\theta r_{\alpha, \theta} * h_{n} * H_{m}\left(e^{\tilde{W}}\right),
\end{aligned}
$$

and so we obtain a.e. in $(0,(1-\eta) \rho)$

$$
\begin{aligned}
h_{n} * H_{m}\left(e^{\tilde{W}}\right) \geq & r_{\alpha, \theta} * \Upsilon_{n, m}-r_{\alpha, \theta} *\left[\tilde{S}_{n} e^{\tilde{W}} H_{m}^{\prime}\left(e^{\tilde{W}}\right)\right] \\
& +\theta h_{n} * r_{\alpha, \theta} * H_{m}\left(e^{\tilde{W}}\right)-\theta r_{\alpha, \theta} * H_{m}\left(e^{\tilde{W}}\right) .
\end{aligned}
$$

Sending $n \rightarrow \infty$ and selecting an appropriate subsequence, if necessary, it follows that

$$
H_{m}\left(e^{\tilde{W}}\right) \geq r_{\alpha, \theta} * \Upsilon_{m}, \quad \text { a.a. } s \in(0,(1-\eta) \rho),
$$

where

$$
\Upsilon_{m}(s)=\int_{0}^{\eta \rho}\left[-\dot{g}_{1-\alpha}(s+\eta \rho-\sigma)\right] H_{m}\left(e^{W(\sigma)}\right) d \sigma .
$$

Observe that for $s \in(0,(1-\eta) \rho)$ we have

$$
0 \leq \theta s^{\alpha} \leq \frac{C_{1}}{r^{2}}(1-\eta)^{\alpha}\left(\tau r^{2 / \alpha}\right)^{\alpha}=C_{1}(1-\eta)^{\alpha} \tau^{\alpha}=: \omega,
$$

and thus by continuity and strict positivity of $E_{\alpha, \alpha}$ in $(-\infty, 0]$,

$r_{\alpha, \theta}(s) \geq \Gamma(\alpha) g_{\alpha}(s) \min _{y \in[0, \omega]} E_{\alpha, \alpha}(-y)=: C_{2}(\alpha, \omega) \Gamma(\alpha) g_{\alpha}(s), \quad s \in(0,(1-\eta) \rho)$.

We may then argue as in $[32$, Section 2.1$]$ to obtain

$$
\begin{array}{r}
H_{m}\left(e^{\tilde{W}(s)}\right) \geq C_{2}(\alpha, \omega) \frac{\alpha(s /[\eta \rho])^{\alpha}}{1+(s /[\eta \rho])}(\eta \rho)^{\alpha-1}\left(g_{1-\alpha} * H_{m}\left(e^{W}\right)\right)(\eta \rho), \\
\text { a.a. } s \in(0,(1-\eta) \rho) .
\end{array}
$$


Evidently, $H_{m}(y) \nearrow y$ as $m \rightarrow \infty$ for all $y \in \mathbb{R}$. Thus by sending $m \rightarrow \infty$ and applying Fatou's lemma we conclude that

$$
\begin{aligned}
& e^{\tilde{W}(s)} \geq C_{2}(\alpha, \omega) \frac{\alpha(s /[\eta \rho])^{\alpha}}{1+(s /[\eta \rho])}(\eta \rho)^{\alpha-1}\left(g_{1-\alpha} * e^{W}\right)(\eta \rho), \\
& \text { a.a. } s \in(0,(1-\eta) \rho) .
\end{aligned}
$$

We then employ (3.50) to estimate as follows.

$$
\begin{aligned}
e^{\lambda} \mu_{1}\left(J_{+}(\lambda)\right) & =e^{\lambda} \mu_{1}\left(\left\{t \in J_{+}: e^{W(t)}<e^{c(u)} e^{-\lambda}\right\}\right)=\int_{J_{+}(\lambda)} e^{\lambda} d t \\
& \leq \int_{J_{+}(\lambda)} e^{c(u)-W(t)} d t \leq \int_{J_{+}} e^{c(u)-W(t)} d t \\
& =\frac{\left(g_{1-\alpha} * e^{W}\right)(\eta \rho)}{g_{2-\alpha}(\eta \rho)} \int_{0}^{(1-\eta) \rho} e^{-\tilde{W}(s)} d s \\
& \leq \frac{C_{2}(\alpha, \omega)^{-1}(\eta \rho)^{1-\alpha}}{\alpha g_{2-\alpha}(\eta \rho)} \int_{0}^{(1-\eta) \rho}(1+s / \eta \rho)(s / \eta \rho)^{-\alpha} d s \\
& =\frac{\Gamma(2-\alpha) \eta \rho}{\alpha C_{2}(\alpha, \omega)} \int_{0}^{\frac{1-\eta}{\eta}} \sigma^{-\alpha}(1+\sigma) d \sigma=C_{3}(\alpha, \eta, \omega) \rho .
\end{aligned}
$$

Hence

$$
I_{4}=\mu_{1}\left(J_{+}(\lambda / 2)\right) \mu_{N}(\delta B) \leq \frac{2 C_{3}(\alpha, \eta, \omega) \delta^{N}}{\lambda} r^{2 / \alpha} \mu_{N}(B), \quad \lambda>0 .
$$

We come now to $I_{1}$. Set $J_{1}(\lambda)=\left\{t \in J_{-}: c-W(t)+\lambda / 2 \geq 0\right\}$ and $\Omega_{t}^{-}(\lambda)=$ $\{x \in \delta B: w(t, x)>c+\lambda\}, t \in J_{1}(\lambda)$, where $c=c(u)$ is given by (3.41). For $t \in J_{1}(\lambda)$, we have

$$
w(t, x)-W(t)>c-W(t)+\lambda \geq \lambda / 2, \quad x \in \Omega_{t}^{-}(\lambda),
$$

and thus we deduce from (3.40) that a.e. in $J_{1}(\lambda)$

$$
\begin{aligned}
& \frac{v}{16 r^{2} \mu_{N}(B)} \mu_{N}\left(\Omega_{t}^{-}(\lambda)\right) \\
& \leq \frac{1}{(c-W+\lambda)^{2}}\left(e^{-W} \partial_{t}\left(g_{1-\alpha, n} * e^{W}\right)+\frac{C_{1}}{r^{2}}+S_{n}\right) .
\end{aligned}
$$

Set $\chi(t, \lambda)=\mu_{N}\left(\Omega_{t}^{-}(\lambda)\right)$, if $t \in J_{1}(\lambda)$, and $\chi(t, \lambda)=0$ in case $t \in J_{-} \backslash J_{1}(\lambda)$. Let further $H(y)=(c-\log y+\lambda)^{-1}, 0<y \leq y_{*}:=e^{c+\lambda / 2}$. Clearly, $H^{\prime}(y)=$ $(c-\log y+\lambda)^{-2} y^{-1}$ as well as

$$
H^{\prime \prime}(y)=\frac{1}{(c-\log y+\lambda)^{2} y^{2}}\left(\frac{2}{c-\log y+\lambda}-1\right), \quad 0<y \leq y_{*},
$$


which shows that $H$ is concave in $\left(0, y_{*}\right]$ whenever $\lambda \geq 4$. We will assume this in what follows.

We next choose a $C^{1}$ extension $\bar{H}$ of $H$ on $(0, \infty)$ such that $\bar{H}$ is concave, $0 \leq \bar{H}^{\prime}(y) \leq \bar{H}^{\prime}\left(y_{*}\right), y_{*} \leq y \leq 2 y_{*}$, and $\bar{H}^{\prime}(y)=0, y \geq 2 y_{*}$. Then

$$
0 \leq y \bar{H}^{\prime}(y) \leq \frac{2}{\lambda}, \quad y>0 .
$$

In fact, for $y \in\left(0, y_{*}\right]$ we have

$$
y \bar{H}^{\prime}(y)=\frac{1}{(c-\log y+\lambda)^{2}} \leq \frac{1}{\left(c-\log y_{*}+\lambda\right)^{2}} \leq \frac{4}{\lambda^{2}} \leq \frac{1}{\lambda},
$$

while in case $y \in\left[y_{*}, 2 y_{*}\right]$ we may simply estimate

$$
y \bar{H}^{\prime}(y) \leq 2 y_{*} \bar{H}^{\prime}\left(y_{*}\right) \leq \frac{2}{\lambda} .
$$

It is clear that $\bar{H}$ is bounded above. There holds

$$
\bar{H}(y) \leq \frac{3}{\lambda}, \quad y>0 .
$$

To see this, note that since $\bar{H}$ is nondecreasing with $\bar{H}^{\prime}(y)=0$ for all $y \geq 2 y_{*}$, the claim follows if the inequality is valid for all $y \in\left[y_{*}, 2 y_{*}\right]$. For such $y$ we have by (3.54) and by concavity of $\bar{H}$

$$
\bar{H}(y) \leq \bar{H}\left(y_{*}\right)+\bar{H}^{\prime}\left(y_{*}\right)\left(y-y_{*}\right) \leq \bar{H}\left(y_{*}\right)+y_{*} \bar{H}^{\prime}\left(y_{*}\right) \leq \frac{3}{\lambda} .
$$

Observe also that

$$
e^{W(t)} H^{\prime}\left(e^{W(t)}\right)=\frac{1}{(c-W(t)+\lambda)^{2}}, \quad \text { a.a. } t \in J_{1}(\lambda) .
$$

Since $\bar{H}^{\prime} \geq 0$, and $e^{-W} \partial_{t}\left(g_{1-\alpha, n} * e^{W}\right)+C_{1} r^{-2}+S_{n} \geq 0$ on $J_{-}$by virtue of (3.40), we infer from (3.52) and (3.53) that

$$
\begin{aligned}
& \frac{v}{16 r^{2} \mu_{N}(B)} \chi(t, \lambda) \\
& \leq e^{W} \bar{H}^{\prime}\left(e^{W}\right)\left(e^{-W} \partial_{t}\left(g_{1-\alpha, n} * e^{W}\right)+\frac{C_{1}}{r^{2}}+S_{n}\right) \\
& \leq \bar{H}^{\prime}\left(e^{W}\right) \partial_{t}\left(g_{1-\alpha, n} * e^{W}\right)+\frac{2 C_{1}}{\lambda r^{2}}+\frac{2\left|S_{n}(t)\right|}{\lambda}, \quad \text { a.a. } t \in J_{-} .
\end{aligned}
$$

Since $\bar{H}$ is concave, the fundamental identity (2.6) yields

$$
\begin{aligned}
\bar{H}^{\prime}\left(e^{W}\right) \partial_{t}\left(g_{1-\alpha, n} * e^{W}\right) & \leq \partial_{t}\left(g_{1-\alpha, n} * \bar{H}\left(e^{W}\right)\right)+\left(-\bar{H}\left(e^{W}\right)+\bar{H}^{\prime}\left(e^{W}\right) e^{W}\right) g_{1-\alpha, n} \\
& \leq \partial_{t}\left(g_{1-\alpha, n} * \bar{H}\left(e^{W}\right)\right)+\frac{2}{\lambda} g_{1-\alpha, n}, \quad \text { a.a. } t \in J_{-},
\end{aligned}
$$


which, together with (3.56), gives a.e. in $J_{-}$

$$
\begin{aligned}
\frac{v}{16 r^{2} \mu_{N}(B)} \chi(t, \lambda) \leq & \partial_{t}\left(\left(g_{1-\alpha, n} * \bar{H}\left(e^{W}\right)\right)\right. \\
& +\frac{2}{\lambda} g_{1-\alpha, n}+\frac{2 C_{1}}{\lambda r^{2}}+\frac{2\left|S_{n}(t)\right|}{\lambda} .
\end{aligned}
$$

We then integrate (3.57) over $J_{-}=(0, \eta \rho)$ and employ (3.55) for the estimate

$$
\left(g_{1-\alpha, n} * \bar{H}\left(e^{W}\right)\right)(\eta \rho) \leq \frac{3}{\lambda} \int_{0}^{\eta \rho} g_{1-\alpha, n}(t) d t .
$$

By sending $n \rightarrow \infty$, this leads to

$$
\begin{aligned}
& \int_{J_{1}(\lambda)} \mu_{N}\left(\Omega_{t}^{-}(\lambda)\right) d t=\int_{0}^{\eta \rho} \chi(t, \lambda) d t \leq \frac{16 r^{2} \mu_{N}(B)}{v}\left(\frac{5}{\lambda} g_{2-\alpha}(\eta \rho)+\frac{2 C_{1} \eta \rho}{\lambda r^{2}}\right) \\
& =\frac{16 r^{2 / \alpha} \mu_{N}(B)}{\nu \lambda}\left(5 g_{2-\alpha}(\eta \tau)+2 C_{1} \eta \tau\right)=: C_{4} \frac{r^{2 / \alpha} \mu_{N}(B)}{\lambda}, \quad \lambda \geq 4 .
\end{aligned}
$$

Hence with $C_{5}=\max \left\{4 \tau, C_{4}\right\}$ we find that

$$
I_{1} \leq \frac{C_{5} r^{2 / \alpha} \mu_{N}(B)}{\lambda}, \quad \lambda>0 .
$$

It remains to derive the desired estimate for $I_{3}$. To this purpose we shift again the time by putting $s=t-\eta \rho$, and denote the corresponding transformed functions as above by $\tilde{W}, \tilde{w}, \ldots$ and so forth. Set further $\tilde{J}_{+}:=(0,(1-\eta) \rho)$. By the timeshifting property (3.2) and by positivity of $e^{W}$, relation (3.40) then implies

$$
\begin{array}{r}
\frac{v}{16 r^{2} \mu_{N}(B)} \int_{\delta B}(\tilde{w}-\tilde{W})^{2} d x \leq e^{-\tilde{W}} \partial_{s}\left(g_{1-\alpha, n} * e^{\tilde{W}}\right)+\frac{C_{1}}{r^{2}}+\tilde{S}_{n}(s), \\
\text { a.a. } s \in \tilde{J}_{+} .
\end{array}
$$

Next, set $J_{2}(\lambda)=\left\{s \in \tilde{J}_{+}: \tilde{W}(s)-c+\lambda / 2 \geq 0\right\}$ and $\Omega_{s}^{+}(\lambda)=\{x \in \delta B:$ $\tilde{w}(s, x)<c-\lambda\}, s \in J_{2}(\lambda)$. For $s \in J_{2}(\lambda)$, we have

$$
\tilde{W}(s)-\tilde{w}(s, x) \geq \tilde{W}(s)-c+\lambda \geq \lambda / 2, \quad x \in \Omega_{s}^{+}(\lambda),
$$

and thus (3.59) yields that a.e. in $J_{2}(\lambda)$

$$
\begin{aligned}
& \frac{v}{16 r^{2} \mu_{N}(B)} \mu_{N}\left(\Omega_{s}^{+}(\lambda)\right) \\
& \leq \frac{1}{(\tilde{W}-c+\lambda)^{2}}\left(e^{-\tilde{W}} \partial_{s}\left(g_{1-\alpha, n} * e^{\tilde{W}}\right)+\frac{C_{1}}{r^{2}}+\tilde{S_{n}}\right) .
\end{aligned}
$$


We proceed now similarly as above for the term $I_{1}$. Set $\chi(s, \lambda)=\mu_{N}\left(\Omega_{s}^{+}(\lambda)\right)$, if $s \in J_{2}(\lambda)$, and $\chi(s, \lambda)=0$ in case $s \in \tilde{J}_{+} \backslash J_{1}(\lambda)$. We consider this time the convex function $H(y)=(\log y-c+\lambda)^{-1}$ for $y \geq y_{*}:=e^{c-\lambda / 2}$ with derivative $H^{\prime}(y)=-(\log y-c+\lambda)^{-2} y^{-1}<0$. We define a $\bar{C}^{1}$ extension $\bar{H}$ of $H$ on $[0, \infty)$ by means of

$$
\bar{H}(y)= \begin{cases}H^{\prime}\left(y_{*}\right)\left(y-y_{*}\right)+H\left(y_{*}\right) & 0 \leq y<y_{*} \\ H(y) & y \geq y_{*}\end{cases}
$$

Evidently, $-\bar{H}$ is concave in $[0, \infty)$ and

$$
0 \leq-\bar{H}^{\prime}(y) y \leq \frac{1}{\left(\log y_{*}-c+\lambda\right)^{2}} \leq \frac{1}{(\lambda / 2)^{2}} \leq \frac{4}{\lambda}, \quad y \geq 0, \lambda \geq 1
$$

We will assume $\lambda \geq 1$ in the subsequent lines.

Observe that

$$
-e^{\tilde{W}(s)} H^{\prime}\left(e^{\tilde{W}(s)}\right)=\frac{1}{(\tilde{W}(s)-c+\lambda)^{2}}, \quad \text { a.a. } s \in J_{2}(\lambda) .
$$

Since $-\bar{H}^{\prime} \geq 0$, and $e^{-\tilde{W}} \partial_{s}\left(g_{1-\alpha, n} * e^{\tilde{W}}\right)+C_{1} r^{-2}+\tilde{S}_{n} \geq 0$ on $\tilde{J}_{+}$due to (3.59), it thus follows from (3.60) and (3.61) that

$$
\begin{aligned}
& \frac{v}{16 r^{2} \mu_{N}(B)} \chi(s, \lambda) \leq-e^{\tilde{W}} \bar{H}^{\prime}\left(e^{\tilde{W}}\right)\left(e^{-\tilde{W}} \partial_{s}\left(g_{1-\alpha, n} * e^{\tilde{W}}\right)+\frac{C_{1}}{r^{2}}+\tilde{S}_{n}\right) \\
& \leq-\bar{H}^{\prime}\left(e^{\tilde{W}}\right) \partial_{s}\left(g_{1-\alpha, n} * e^{\tilde{W}}\right)+\frac{4 C_{1}}{\lambda r^{2}}+\frac{4\left|\tilde{S}_{n}(s)\right|}{\lambda}, \quad \text { a.a. } s \in \tilde{J}_{+} .
\end{aligned}
$$

By concavity of $-\bar{H}$, the fundamental identity (2.6) provides the estimate

$$
\begin{aligned}
& -\bar{H}^{\prime}\left(e^{\tilde{W}}\right) \partial_{s}\left(g_{1-\alpha, n} * e^{\tilde{W}}\right) \leq-\partial_{s}\left(g_{1-\alpha, n} * \bar{H}\left(e^{\tilde{W}}\right)\right)+\left(\bar{H}\left(e^{\tilde{W}}\right)-\bar{H}^{\prime}\left(e^{\tilde{W}}\right) e^{\tilde{W}}\right) g_{1-\alpha, n} \\
& \leq-\partial_{s}\left(g_{1-\alpha, n} * \bar{H}\left(e^{\tilde{W}}\right)\right)+\bar{H}(0) g_{1-\alpha, n} \leq-\partial_{s}\left(g_{1-\alpha, n} * \bar{H}\left(e^{\tilde{W}}\right)\right)+\frac{6}{\lambda} g_{1-\alpha, n},
\end{aligned}
$$

a.e. in $\tilde{J}_{+}$, which when combined with (3.62) leads to

$$
\frac{v}{16 r^{2} \mu_{N}(B)} \chi(s, \lambda) \leq-\partial_{s}\left(g_{1-\alpha, n} * \bar{H}\left(e^{\tilde{W}}\right)\right)+\frac{6}{\lambda} g_{1-\alpha, n}+\frac{4 C_{1}}{\lambda r^{2}}+\frac{4\left|\tilde{S}_{n}(s)\right|}{\lambda}
$$


for a.a. $s \in \tilde{J}_{+}$. We integrate this estimate over $\tilde{J}_{+}$and send $n \rightarrow \infty$ to the result

$$
\begin{aligned}
\int_{J_{2}(\lambda)} \mu_{N}\left(\Omega_{s}^{+}(\lambda)\right) d s & =\int_{0}^{(1-\eta) \rho} \chi(s, \lambda) d s \\
& \leq \frac{16 r^{2} \mu_{N}(B)}{v}\left(\frac{6}{\lambda} g_{2-\alpha}((1-\eta) \rho)+\frac{4 C_{1}(1-\eta) \rho}{\lambda r^{2}}\right) \\
& =\frac{16 r^{2 / \alpha} \mu_{N}(B)}{\nu \lambda}\left(6 g_{2-\alpha}((1-\eta) \tau)+4 C_{1}(1-\eta) \tau\right) \\
& =: C_{6} \frac{r^{2 / \alpha} \mu_{N}(B)}{\lambda}, \quad \lambda \geq 1 .
\end{aligned}
$$

Hence with $C_{7}=\max \left\{\tau, C_{6}\right\}$ we obtain that

$$
I_{3} \leq \frac{C_{7} r^{2 / \alpha} \mu_{N}(B)}{\lambda}, \quad \lambda>0 .
$$

Finally, combining (3.42), (3.43), and (3.44), (3.51), (3.58), (3.63) establishes the theorem.

\subsection{The final step}

We are now in position to prove Theorem 1.1. Without loss of generality we may assume that $u \geq \varepsilon$ for some $\varepsilon>0$; otherwise replace $u$ by $u+\varepsilon$, which is a supersolution of (1.1) with $u_{0}+\varepsilon$ instead of $u_{0}$, and eventually let $\varepsilon \rightarrow 0+$.

For $0<\sigma \leq 1$, we set $U_{\sigma}=\left(t_{0}+(2-\sigma) \tau r^{2 / \alpha}, t_{0}+2 \tau r^{2 / \alpha}\right) \times \sigma B$ and $U_{\sigma}^{\prime}=\left(t_{0}, t_{0}+\sigma \tau r^{2 / \alpha}\right) \times \sigma B$. Clearly, $Q_{-}\left(t_{0}, x_{0}, r\right)=U_{\delta}^{\prime}$ and $Q_{+}\left(t_{0}, x_{0}, r\right)=U_{\delta}$.

By Theorem 3.2,

$$
\underset{U_{\sigma^{\prime}}}{\operatorname{ess} \sup } u^{-1} \leq\left(\frac{C \mu_{N+1}\left(U_{1}\right)^{-1}}{\left(\sigma-\sigma^{\prime}\right)^{\tau_{0}}}\right)^{1 / \gamma}\left|u^{-1}\right|_{L_{\gamma}\left(U_{\sigma}\right)}, \quad \delta \leq \sigma^{\prime}<\sigma \leq 1, \quad \gamma \in(0,1]
$$

Here $C=C(\nu, \Lambda, \delta, \tau, \alpha, N)$ and $\tau_{0}=\tau_{0}(\alpha, N)$. This shows that the first hypothesis of Lemma 2.3 is satisfied by any positive constant multiple of $u^{-1}$ with $\beta_{0}=\infty$.

Consider now $f_{1}=u^{-1} e^{c(u)}$ where $c(u)$ is the constant from Theorem 3.4 with $K_{-}=U_{1}^{\prime}$ and $K_{+}=U_{1}$. Since $\log f_{1}=c(u)-\log u$, we see from Theorem 3.4, estimate (3.32), that

$$
\mu_{N+1}\left(\left\{(t, x) \in U_{1}: \log f_{1}(t, x)>\lambda\right\}\right) \leq M \mu_{N+1}\left(U_{1}\right) \lambda^{-1}, \quad \lambda>0,
$$

where $M=M(\nu, \Lambda, \delta, \tau, \eta, \alpha, N)$. Hence we may apply Lemma 2.3 with $\beta_{0}=\infty$ to $f_{1}$ and the family $U_{\sigma}$; thereby we obtain

$$
\underset{U_{\delta}}{\operatorname{ess} \sup } f_{1} \leq M_{1}
$$


with $M_{1}=M_{1}(\nu, \Lambda, \delta, \tau, \eta, \alpha, N)$. In terms of $u$ this means that

$$
e^{c(u)} \leq M_{1} \underset{U_{\delta}}{\operatorname{ess} \inf } u
$$

On the other hand, Theorem 3.3 yields

$$
|u|_{L_{p}\left(U_{\sigma^{\prime}}^{\prime}\right)} \leq\left(\frac{C \mu_{N+1}\left(U_{1}^{\prime}\right)^{-1}}{\left(\sigma-\sigma^{\prime}\right)^{\tau_{1}}}\right)^{1 / \gamma-1 / p}|u|_{L_{\gamma}\left(U_{\sigma}^{\prime}\right)}, \quad \delta \leq \sigma^{\prime}<\sigma \leq 1,0<\gamma \leq p / \tilde{\kappa} .
$$

Here $C=C(\nu, \Lambda, \delta, \tau, \alpha, N, p)$ and $\tau_{1}=\tau_{1}(\alpha, N)$. Thus the first hypothesis of Lemma 2.3 is satisfied by any positive constant multiple of $u$ with $\beta_{0}=p$ and $\eta=1 / \tilde{\kappa}$. Taking $f_{2}=u e^{-c(u)}$ with $c(u)$ from above, we have $\log f_{2}=\log u-c(u)$ and so Theorem 3.4, estimate (3.31), gives

$$
\mu_{N+1}\left(\left\{(t, x) \in U_{1}^{\prime}: \log f_{2}(t, x)>\lambda\right\}\right) \leq M \mu_{N+1}\left(U_{1}^{\prime}\right) \lambda^{-1}, \quad \lambda>0,
$$

where $M$ is as above. Therefore we may again apply Lemma 2.3 , this time to the function $f_{2}$ and the sets $U_{\sigma}^{\prime}$, and with $\beta_{0}=p$ and $\eta=1 / \tilde{\kappa}$; we get

$$
\left|f_{2}\right|_{L_{p}\left(U_{\delta}^{\prime}\right)} \leq M_{2} \mu_{N+1}\left(U_{1}^{\prime}\right)^{1 / p}
$$

where $M_{2}=M_{2}(\nu, \Lambda, \delta, \tau, \eta, \alpha, N, p)$. Rephrasing then yields

$$
\mu_{N+1}\left(U_{1}^{\prime}\right)^{-1 / p}|u|_{L_{p}\left(U_{\delta}^{\prime}\right)} \leq M_{2} e^{c(u)} .
$$

Finally, we combine (3.64) and (3.65) to the result

$$
\mu_{N+1}\left(U_{1}^{\prime}\right)^{-1 / p}|u|_{L_{p}\left(U_{\delta}^{\prime}\right)} \leq M_{1} M_{2} \underset{U_{\delta}}{\operatorname{essinf}} u
$$

which proves the assertion.

\section{Optimality of the exponent $\frac{2+N \alpha}{2+N \alpha-2 \alpha}$ in the weak Harnack inequality}

In this section we will show that the exponent $\frac{2+N \alpha}{2+N \alpha-2 \alpha}$ in Theorem 1.1 is optimal. on $\mathbb{R}^{N}$

To this purpose consider the nonhomogeneous fractional diffusion equation

$$
\partial_{t}^{\alpha} u-\Delta u=f, \quad t \in(0, T], x \in \mathbb{R}^{N},
$$

with initial condition

$$
u(0, x)=0, \quad x \in \mathbb{R}^{n} .
$$


Following [10], we say that a function $u \in C\left([0, T] \times \mathbb{R}^{N}\right) \cap C\left((0, T] ; C^{2}\left(\mathbb{R}^{N}\right)\right)$ with $g_{1-\alpha} * u \in C^{1}\left((0, T] ; C\left(\mathbb{R}^{N}\right)\right)$ is a classical solution of the problem (4.1), (4.2) if $u$ satisfies (4.1) and (4.2). For any bounded continuous function $f$ that is locally Hölder continuous in $x$, there exists a unique classical solution $u$ of the problem (4.1), (4.2), and it is of the form

$$
u(t, x)=\int_{0}^{t} \int_{\mathbb{R}^{N}} Y(t-\tau, x-y) f(\tau, y) d y d \tau,
$$

where

$$
Y(t, x)=c(N)|x|^{-N} t^{\alpha-1} H_{12}^{20}\left(\frac{1}{4} t^{-\alpha}|x|^{2} \mid \begin{array}{l}
(\alpha, \alpha) \\
(N / 2,1),(1,1)
\end{array}\right),
$$

$c f$. [10]. Here $H_{12}^{20}\left(\left.z\right|_{(N / 2,1),(1,1)} ^{(\alpha, \alpha)}\right)$ denotes a special $H$ function (also termed Fox's $H$ function), see [17, Section 1.12] and [10] for its definition. It is differentiable for $z>0$, the asymptotic behaviour for $z \rightarrow \infty$ and $z \rightarrow+0$, respectively, is described in [10, formulae (3.9) and (3.14)]. It has been also proved in [10] that $Y$ is nonnegative.

We choose a smooth and nonnegative approximation of unity $\left\{\phi_{n}(t, x)\right\}_{n \in \mathbb{N}}$ in $\mathbb{R}_{+} \times \mathbb{R}^{N}$ such that each $\phi_{n}$ is bounded. Put $f=\phi_{n}$ in (4.1) and denote the corresponding classical solution of (4.1), (4.2) by $u_{n}$. Evidently, $u_{n}$ is nonnegative and satisfies

$$
\partial_{t}^{\alpha} u_{n}-\Delta u_{n}=\phi_{n} \geq 0, \quad t \in(0, T], x \in \mathbb{R}^{N} .
$$

Hence $u_{n}$ is a nonnegative supersolution of (4.1) with $f=0$ for all $n \in \mathbb{N}$.

Suppose the weak Harnack inequality (1.3) holds for some $p \geq \frac{2+N \alpha}{2+N \alpha-2 \alpha}$. Then, by taking $Q_{-}=(0,1) \times B(0,1)$ and $Q_{+}=(2,3) \times B(0,1)$ it follows that

$$
\left(\int_{Q_{-}} u_{n}^{p} d \mu_{N+1}\right)^{1 / p} \leq C \inf _{Q_{+}} u_{n}, \quad n \in \mathbb{N},
$$

where the constant $C$ is independent of $n$. Since $u_{n} \rightarrow Y$ in the distributional sense as $n \rightarrow \infty$, we have

$$
\begin{aligned}
\inf _{Q_{+}} u_{n} & \leq \frac{1}{\mu_{N+1}\left(Q_{+}\right)} \int_{Q_{+}} u_{n} d \mu_{N+1} \\
& \leq 1+\frac{1}{\mu_{N+1}\left(Q_{+}\right)} \int_{Q_{+}} Y d \mu_{N+1}<\infty, \quad n \geq n_{0},
\end{aligned}
$$

for a sufficiently large $n_{0}$. On the other hand, the left-hand side of (4.4) cannot stay bounded, since $Y \notin L_{p}\left(Q_{-}\right)$for $p \geq \frac{2+N \alpha}{2+N \alpha-2 \alpha}$. In fact, writing $H_{12}^{20}(z)=$ 
$H_{12}^{20}\left(\left.z\right|_{(N / 2,1),(1,1)} ^{(\alpha, \alpha)}\right)$ for short, we have

$$
\begin{aligned}
|Y|_{L_{p}\left(Q_{-}\right)}^{p} & =\int_{0}^{1} \int_{B(0,1)} c(N)^{p}|x|^{-N p} t^{(\alpha-1) p} H_{12}^{20}\left(t^{-\alpha}|x|^{2} / 4\right)^{p} d x d t \\
& =c_{1} \int_{0}^{1} \int_{0}^{1} r^{N-1-N p} t^{(\alpha-1) p} H_{12}^{20}\left(t^{-\alpha} r^{2} / 4\right)^{p} d r d t \\
& =c_{1} \int_{0}^{1} \int_{0}^{t^{-\alpha / 2}}\left(\rho t^{\alpha / 2}\right)^{N-1-N p} t^{(\alpha-1) p+\alpha / 2} H_{12}^{20}\left(\rho^{2} / 4\right)^{p} d \rho d t \\
& \geq c_{1} \int_{0}^{1} t^{\alpha(N-N p) / 2+(\alpha-1) p} d t \int_{0}^{1} \rho^{N-1-N p} H_{12}^{20}\left(\rho^{2} / 4\right)^{p} d \rho \\
& \geq c_{2} \int_{0}^{1} t^{\alpha(N-N p) / 2+(\alpha-1) p} d t,
\end{aligned}
$$

with some positive constant $c_{2}$. The last integral diverges for all $p \geq \frac{2+N \alpha}{2+N \alpha-2 \alpha}$. Hence (4.4) yields a contradiction.

\section{Applications of the weak Harnack inequality}

The strong maximum principle for weak subsolutions of (1.1) may be easily derived as a consequence of the weak Harnack inequality.

Theorem 5.1. Let $\alpha \in(0,1), T>0$, and $\Omega \subset \mathbb{R}^{N}$ be a bounded domain. Suppose the assumptions $(\mathrm{H} 1)-(\mathrm{H} 3)$ are satisfied. Let $u \in Z_{\alpha}$ be a weak subsolution of (1.1)

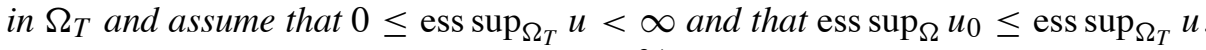
Then, if for some cylinder $Q=\left(t_{0}, t_{0}+\tau r^{2 / \alpha}\right) \times B\left(x_{0}, r\right) \subset \Omega_{T}$ with $t_{0}, \tau, r>0$ and $\overline{B\left(x_{0}, r\right)} \subset \Omega$ we have

$$
\underset{Q}{\operatorname{ess} \sup } u=\underset{\Omega_{T}}{\operatorname{ess} \sup } u,
$$

the function $u$ is constant on $\left(0, t_{0}\right) \times \Omega$.

Proof. Let $M=\operatorname{ess} \sup _{\Omega_{T}} u$. Then $v:=M-u$ is a nonnegative weak supersolution of (1.1) with $u_{0}$ replaced by $v_{0}:=M-u_{0} \geq 0$. For any $0 \leq t_{1}<t_{1}+\eta r^{2 / \alpha}<t_{0}$ the weak Harnack inequality with $p=1$ applied to $v$ yields an estimate of the form

$$
r^{-(N+2 / \alpha)} \int_{t_{1}}^{t_{1}+\eta r^{2 / \alpha}} \int_{B\left(x_{0}, r\right)}(M-u) d x d t \leq C \underset{Q}{\operatorname{essinf}(M-u)=0 .}
$$

This shows that $u=M$ a.e. in $\left(0, t_{0}\right) \times B\left(x_{0}, r\right)$. As in the classical parabolic case (cf. [19]) the assertion now follows by a chaining argument. 
We next apply the weak Harnack inequality to establish continuity at $t=0$ for weak solutions.

Theorem 5.2. Let $\alpha \in(0,1), T>0$, and $\Omega \subset \mathbb{R}^{N}$ be a bounded domain. Suppose the assumptions $(\mathrm{H} 1)$ and $(\mathrm{H} 2)$ are satisfied. Let $u \in Z_{\alpha}$ be a bounded weak solution of (1.1) in $\Omega_{T}$ with $u_{0}=0$. Then $u$ is continuous at $\left(0, x_{0}\right)$ for all $x_{0} \in \Omega$ and $\lim _{(t, x) \rightarrow\left(0, x_{0}\right)} u(t, x)=0$. Moreover, letting $\eta>0$ we have for any cylinder $Q\left(x_{0}, r_{0}\right):=\left(0, \eta r_{0}^{2 / \alpha}\right) \times B\left(x_{0}, r_{0}\right) \subset \Omega_{T}$ and $r \in\left(0, r_{0}\right]$

$$
\underset{Q\left(x_{0}, r\right)}{\operatorname{ess} \operatorname{osc}} u \leq C\left(\frac{r}{r_{0}}\right)^{\delta}|u|_{L_{\infty}\left(\Omega_{T}\right)}
$$

with

$$
\underset{Q\left(x_{0}, r\right)}{\operatorname{ess} \text { osc }}=\underset{Q\left(x_{0}, r\right)}{\operatorname{esss}}-\underset{Q\left(x_{0}, r\right)}{\operatorname{essinf}}
$$

and constants $C=C f(\nu, \Lambda, \eta, \alpha, N)>0$ and $\delta=\delta(\nu, \Lambda, \eta, \alpha, N) \in(0,1)$.

Proof. Let $u \in Z_{\alpha}$ be a bounded weak solution of (1.1) in $\Omega_{T}$ with $u_{0}=0$. Set $u(t, x)=0$ and $A(t, x)=I d$ for $t<0$ and $x \in \Omega$. For $T_{0}>0$ we shift the time by setting $s=t+T_{0}$ and put $\tilde{f}(s)=f\left(s-T_{0}\right), s \in\left(0, T+T_{0}\right)$, for functions $f$ defined on $\left(-T_{0}, T\right)$. Since $D u(t, \cdot)=0$ for $t<0$ and

$$
\partial_{t}\left(g_{1-\alpha, n} * u\right)(t, x)=\partial_{t} \int_{-T_{0}}^{t} g_{1-\alpha, n}(t-\tau) u(\tau, x) d \tau=\partial_{s}\left(g_{1-\alpha, n} * \tilde{u}\right)(s, x),
$$

the function $\tilde{u}$ is a bounded weak solution of

$$
\partial_{s}^{\alpha} \tilde{u}-\operatorname{div}(\tilde{A}(s, x) D \tilde{u})=0, \quad s \in\left(0, T+T_{0}\right), x \in \Omega .
$$

Next, assuming $r \in\left(0, r_{0} / 2\right]$ we introduce the cylinders

$$
\begin{aligned}
Q_{*}\left(x_{0}, r\right) & =\left(-\eta r^{2 / \alpha}, \eta r^{2 / \alpha}\right) \times B\left(x_{0}, r\right), \\
Q_{-}\left(x_{0}, r\right) & =\left(-\eta(2 r)^{2 / \alpha},-\eta(3 r / 2)^{2 / \alpha}\right) \times B\left(x_{0}, r\right),
\end{aligned}
$$

and denote by $\tilde{Q}_{*}\left(x_{0}, r\right)$ resp. $\tilde{Q}_{-}\left(x_{0}, r\right)$ the corresponding cylinders in the $(s, x)$ coordinate system. Let us write $M_{i}=\operatorname{ess} \sup _{\tilde{Q}_{*}\left(x_{0}, i r\right)} \tilde{u}$ and $m_{i}=\operatorname{essinf}_{\tilde{Q}_{*}\left(x_{0}, i r\right)} \tilde{u}$ for $i=1,2$. Choosing $T_{0} \geq \eta(2 r)^{2 / \alpha}$, we may apply Theorem 1.1 with $p=1$ to the functions $M_{2}-\tilde{u}, \tilde{u}-m_{2}$, which are nonnegative in $\left(0, \eta(2 r)^{2 / \alpha}+T_{0}\right) \times B\left(x_{0}, 2 r\right)$, thereby obtaining

$$
\begin{aligned}
& r^{-N+2 / \alpha} \int_{\tilde{Q}_{-}\left(x_{0}, r\right)}\left(M_{2}-\tilde{u}\right) d \mu_{N+1} \leq C\left(M_{2}-M_{1}\right), \\
& r^{-N+2 / \alpha} \int_{\tilde{Q}_{-}\left(x_{0}, r\right)}\left(\tilde{u}-m_{2}\right) d \mu_{N+1} \leq C\left(m_{1}-m_{2}\right),
\end{aligned}
$$


where $C>1$ is a constant independent of $u$ and $r$. By addition, it follows that

$$
M_{2}-m_{2} \leq C\left(M_{2}-m_{2}+m_{1}-M_{1}\right) .
$$

Writing $\omega\left(x_{0}, r\right)=\operatorname{ess}_{\sup } \tilde{Q}_{*}\left(x_{0}, i r\right) \tilde{u}-\operatorname{ess} \inf _{\tilde{Q}_{*}\left(x_{0}, i r\right)} \tilde{u}$, this yields

$$
\omega\left(x_{0}, r\right) \leq \theta \omega\left(x_{0}, 2 r\right), \quad r \leq r_{0} / 2,
$$

where $\theta=1-C^{-1} \in(0,1)$. Iterating (5.3) as in the proof of [11, Lemma 8.23] we obtain

$$
\omega\left(x_{0}, r\right) \leq \frac{1}{\theta}\left(\frac{r}{r_{0}}\right)^{\log \theta / \log (1 / 2)} \omega\left(x_{0}, r_{0}\right), \quad r \leq r_{0} .
$$

The estimate (5.2) then follows by transforming back to the function $u$ and using that $u=0$ for negative times. In particular, we also see that $u$ is continuous at $\left(0, x_{0}\right)$ for all $x_{0} \in \Omega$ and that $\lim _{(t, x) \rightarrow\left(0, x_{0}\right)} u(t, x)=0$.

The last application is a uniqueness theorem for global bounded weak solutions. We say that a function $u$ on $\mathbb{R}_{+} \times \mathbb{R}^{N}$ is a global weak solution of

$$
\partial_{t}^{\alpha} u-\operatorname{div}(A(t, x) D u)=0,
$$

if it is a weak solution of (5.4) in $(0, T) \times B(0, r)$ for all $T>0$ and $r>0$.

Corollary 5.3. Let $\alpha \in(0,1)$. Assume that $A \in L_{\infty}\left(\mathbb{R}_{+} \times \mathbb{R}^{N} ; \mathbb{R}^{N \times N}\right)$ and that there exists $v>0$ such that

$$
(A(t, x) \xi \mid \xi) \geq v|\xi|^{2}, \quad \text { for a.a. }(t, x) \in \mathbb{R}_{+} \times \mathbb{R}^{N} \text {, and all } \xi \in \mathbb{R}^{N} .
$$

Suppose that $u$ is a global bounded weak solution of (5.4). Then $u=0$ a.e. on $\mathbb{R}_{+} \times \mathbb{R}^{N}$.

Proof. For $r>0$ and $x_{0}=0$ it follows from the proof of Theorem 5.2 that

$$
\omega(0, r) \leq \theta \omega(0,2 r), \quad r>0,
$$

where $\theta \in(0,1)$ is independent of $r$ and $u$. By induction, (5.5) yields

$$
\omega(0, r) \leq \theta^{n} \omega\left(0,2^{n} r\right) \leq 2 \theta^{n}|u|_{L_{\infty}\left(\mathbb{R}_{+} \times \mathbb{R}^{N}\right)}, \quad r>0, n \in \mathbb{N} .
$$

Sending $n \rightarrow \infty$ shows that $u$ is constant. The claim then follows by Theorem 5.2. 


\section{References}

[1] E. BAZHLEKova, "Fractional Evolution Equations in Banach Spaces", Dissertation, Technische Universiteit Eindhoven, 2001.

[2] E. BombIERI and E. GIUSTI, Harnack's inequality for elliptic differential equations on minimal surfaces, Invent. Math. 15 (1972), 24-46.

[3] Ph. ClÉment, On abstract Volterra equations in Banach spaces with completely positive kernels, In: "Infinite-dimensional systems" (Retzhof, 1983), Lecture Notes in Math., Vol. 1076, Springer, Berlin, 1984, 32-40.

[4] Ph. Clément, S.-O. Londen and G. Simonett, Quasilinear evolutionary equations and continuous interpolation spaces, J. Differential Equations 196 (2004), 418-447.

[5] PH. ClÉment and J. A. NoHel, Asymptotic behavior of solutions of nonlinear Volterra equations with completely positive kernels, SIAM J. Math. Anal. 12 (1981), 514-534.

[6] PH. ClÉment and J. PRÜSS, Completely positive measures and Feller semigroups, Math. Ann. 287 (1990), 73-105.

[7] Ph. ClÉment and J. PrÜss, Global existence for a semilinear parabolic Volterra equation, Math. Z. 209 (1992), 17-26.

[8] PH. ClÉMENT and R. ZACHER, A priori estimates for weak solutions of elliptic equations, Technical Report, Martin-Luther University Halle-Wittenberg, Germany, 2004.

[9] E. DiBenedetto, "Degenerate Parabolic Equations", Springer, New York, 1993.

[10] S. E. EIDELMAN and A. N. KoCHUBEI, Cauchy problem for fractional diffusion equations, J. Differential Equations 199 (2004), 211-255.

[11] D. Gilbarg and N. Trudinger, "Elliptic Partial Differential Equations of Second Order", Springer, 1977.

[12] G. GRIPENBERG, Volterra integro-differential equations with accretive nonlinearity, J. Differential Equations 60 (1985), 57-79.

[13] G. Gripenberg, S.-O. Londen and O. Staffans, "Volterra Integral and Functional Equations", In: Encyclopedia of Mathematics and its Applications, 34, Cambridge University Press, Cambridge, 1990.

[14] R. HiLfer, Fractional time evolution, In: "Applications of Fractional Calculus in Physics", R. Hilfer (ed.), World Sci. Publ., River Edge, NJ, 2000, 87-130.

[15] R. HiLfer, On fractional diffusion and continuous time random walks, Phys. A 329 (2003), $35-40$.

[16] M. KASSMANn, The classical Harnack inequality fails for non-local operators, SFB 611preprint no. 360, University of Bonn, Germany, 2007.

[17] A. A. Kilbas, H. M. Srivastava and J. J. Trujillo, "Theory and Applications of Fractional Differential Equations", Elsevier, 2006.

[18] O. A. Ladyzenskaja, V. A. Solonnikov and N. N. Uralceva, "Linear and Quasilinear Equations of Parabolic Type", Translations of Mathematical Monographs, Vol. 23, American Mathematical Society, Providence, R.I., 1968.

[19] G. M. Lieberman, "Second Order Parabolic Differential Equations", World Scientific, London, 1996.

[20] R. METZLER and J. KLAFTER, The random walk's guide to anomalous diffusion: a fractional dynamics approach, Phys. Rep. 339 (2000), 1-77.

[21] J. MOSER, A new proof of De Giorgi's theorem concerning the regularity problem for elliptic differential equations, Comm. Pure Appl. Math. 13 (1960), 457-468.

[22] J. Moser, A Harnack inequality for parabolic differential equations, Comm. Pure Appl. Math. 17 (1964), 101-134; correction in Comm. Pure Appl. Math. 20 (1967), 231-236.

[23] J. Moser, On a pointwise estimate for parabolic differential equations, Comm. Pure Appl. Math. 24 (1971), 727-740.

[24] J. PRÜSS, "Evolutionary Integral Equations and Applications", Monographs in Mathematics, Vol. 87, Birkhäuser, Basel, 1993. 
[25] H. E. Roman and P. A. Alemany, Continuous-time random walks and the fractional diffusion equation, J. Phys. A 27 (1994), 3407-3410.

[26] L. S ALOFF-CosTE, "Aspects of Sobolev-Type Inequalities", London Mathematical Society Lecture Note Series, Vol. 289, University Press, Cambridge, 2002.

[27] E. Scalas, R. Gorenflo and F. Mainard, Fractional calculus and continuous-time finance, Phys. A 284 (2000), 376-384.

[28] L. SILVESTRE, Hölder estimates for solutions of integro-differential equations like the fractional Laplace, Indiana Univ. Math. J. 55 (2006), 1155-1174.

[29] N. S. TRUDINGER, Pointwise estimates and quasilinear parabolic equations, Comm. Pure Appl. Math. 21 (1968), 205-226.

[30] V. VERGARA and R. ZACHER, Lyapunov functions and convergence to steady state for differential equations of fractional order, Math. Z. 259 (2008), 287-309.

[31] R. ZACHER, A De Giorgi-Nash type theorem for time fractional diffusion equations, Math. Ann. 356 (2013), 99-146.

[32] R. ZACHER, A weak Harnack inequality for fractional differential equations, J. Integral Equations Appl. 19 (2007), 209-232.

[33] R. ZACHER, Boundedness of weak solutions to evolutionary partial integro-differential equations with discontinuous coefficients, J. Math. Anal. Appl. 348 (2008), 137-149.

[34] R. ZACHER, Maximal regularity of type $L_{p}$ for abstract parabolic Volterra equations, J. Evol. Equ. 5 (2005), 79-103.

[35] R. ZACHER, Quasilinear parabolic integro-differential equations with nonlinear boundary conditions, Differential Integral Equations 19 (2006), 1129-1156.

[36] R. ZACHER, The Harnack inequality for the Riemann-Liouville fractional derivation operator, Math. Inequal. Appl. 14 (2011), 35-43.

[37] R. ZACHER, Weak solutions of abstract evolutionary integro-differential equations in Hilbert spaces, Funkcial. Ekvac. 52 (2009), 1-18.

Martin-Luther University

Halle-Wittenberg

Institute of Mathematics

Theodor-Lieser-Strasse 5

06120 Halle, Germany,

rico.zacher@mathematik.uni-halle.de 